\title{
A Cosmological Model Based on a Quadratic Equation of State Unifying Vacuum Energy, Radiation, and Dark Energy
}

\author{
Pierre-Henri Chavanis \\ Laboratoire de Physique Théorique, IRSAMC, CNRS, UPS, Université de Toulouse, 31062 Toulouse, France \\ Correspondence should be addressed to Pierre-Henri Chavanis; chavanis@irsamc.ups-tlse.fr
}

Received 26 March 2013; Accepted 17 May 2013

Academic Editor: Kazuharu Bamba

Copyright (C) 2013 Pierre-Henri Chavanis. This is an open access article distributed under the Creative Commons Attribution License, which permits unrestricted use, distribution, and reproduction in any medium, provided the original work is properly cited.

We consider a cosmological model based on a quadratic equation of state $p / c^{2}=-4 \rho^{2} / 3 \rho_{P}+\rho / 3-4 \rho_{\Lambda} / 3$ (where $\rho_{P}$ is the Planck density and $\rho_{\Lambda}$ is the cosmological density) "unifying" vacuum energy, radiation, and dark energy. For $\rho \rightarrow \rho_{P}$, it reduces to $p=-\rho c^{2}$ leading to a phase of early accelerated expansion (early inflation) with a constant density equal to the Planck density $\rho_{P}=5.16 \cdot 10^{99} \mathrm{~g} / \mathrm{m}^{3}$ (vacuum energy). For $\rho_{\Lambda} \ll \rho \ll \rho_{P}$, we recover the equation of state of radiation $p=\rho c^{2} / 3$. For $\rho \rightarrow \rho_{\Lambda}$, we get $p=-\rho c^{2}$ leading to a phase of late accelerated expansion (late inflation) with a constant density equal to the cosmological density $\rho_{\Lambda}=7.02 \cdot 10^{-24} \mathrm{~g} / \mathrm{m}^{3}$ (dark energy). The temperature is determined by a generalized Stefan-Boltzmann law. We show a nice "symmetry" between the early universe (vacuum energy + radiation) and the late universe (radiation + dark energy). In our model, they are described by two polytropic equations of state with index $n=+1$ and $n=-1$, respectively. Furthermore, the Planck density $\rho_{P}$ in the early universe plays a role similar to that of the cosmological density $\rho_{\Lambda}$ in the late universe. They represent fundamental upper and lower density bounds differing by 122 orders of magnitude. We add the contribution of baryonic matter and dark matter considered as independent species and obtain a simple cosmological model describing the whole evolution of the universe. We study the evolution of the scale factor, density, and temperature. This model gives the same results as the standard $\Lambda$ CDM model for $t>23.3 t_{P}$, where $t_{P}=5.39 \cdot 10^{-44} \mathrm{~s}$ is the Planck time and completes it by incorporating the phase of early inflation in a natural manner. Furthermore, this model does not present any singularity at $t=0$ and exists eternally in the past (although it may be incorrect to extrapolate the solution to the infinite past). Our study suggests that vacuum energy, radiation, and dark energy may be the manifestation of a unique form of "generalized radiation." By contrast, the baryonic and dark matter components of the universe are treated as different species. This is at variance with usual models (quintessence, Chaplygin gas, ...) trying to unify dark matter and dark energy.

\section{Introduction}

The evolution of the universe may be divided into four main periods [1]. In the vacuum energy era (Planck era), the universe undergoes a phase of early inflation that brings it from the Planck size $l_{P}=1.62 \cdot 10^{-35} \mathrm{~m}$ to an almost "macroscopic" size $a \sim 10^{-6} \mathrm{~m}$ in a tiniest fraction of a second $[2-5]$. The universe then enters in the radiation era and, when the temperature cools down below approximately $10^{3} \mathrm{~K}$, in the matter era [6]. Finally, in the dark energy era (de Sitter era), the universe undergoes a phase of late inflation [7]. The early inflation is necessary to solve notorious difficulties such as the singularity problem, the flatness problem, and the horizon problem [2-5]. The late inflation is necessary to account for the observed accelerating expansion of the universe [8-11]. At present, the universe is composed of approximately $5 \%$ baryonic matter, 20\% dark matter, and 75\% dark energy [1]. Despite the success of the standard model, the nature of vacuum energy, dark matter, and dark energy remains very mysterious and leads to many speculations.

The phase of inflation in the early universe is usually described by some hypothetical scalar field $\phi$ with its origin in quantum fluctuations of vacuum [2-5]. This leads to an equation of state $p=-\rho c^{2}$, implying a constant energy density, called the vacuum energy. This energy density is usually identified with the Planck density $\rho_{P}=5.16 \cdot 10^{99} \mathrm{~g} / \mathrm{m}^{3}$. 
As a result of the vacuum energy, the universe expands exponentially rapidly on a timescale of the order of the Planck time $t_{P}=5.39 \cdot 10^{-44} \mathrm{~s}$. This phase of early inflation is followed by the radiation era described by an equation of state $p=\rho c^{2} / 3$. In the vacuum energy era, the density has a constant value $\rho \simeq \rho_{P}$, while it decreases as $\rho \propto a^{-4}$ during the radiation era.

The phase of acceleration in the late universe is usually ascribed to the cosmological constant $\Lambda$ which is equivalent to a constant energy density $\rho_{\Lambda}=\Lambda /(8 \pi G)=7.02 \cdot 10^{-24} \mathrm{~g} / \mathrm{m}^{3}$ called the dark energy. This acceleration can be modeled by an equation of state $p=-\rho c^{2}$, implying a constant energy density identified with the cosmological density $\rho_{\Lambda}$. As a result of the dark energy, the universe expands exponentially rapidly on a timescale of the order of the cosmological time $t_{\Lambda}=1.46 \cdot 10^{18} \mathrm{~s}$ (de Sitter solution). This leads to a phase of late inflation. Inspired by the analogy with the early inflation, some authors have represented the dark energy by a scalar field called quintessence [12-24]. As an alternative to the quintessence, other authors have proposed to model the acceleration of the universe by an exotic fluid with an equation of state of the form $p / c^{2}=B \rho-A / \rho^{a}$ with $A \geq 0$ and $a \geq-1$ called the Chaplygin gas when $(B, a)=(0,1)$ and the generalized Chaplygin gas otherwise [25-32]. This equation of state may be rewritten as

$$
p=\left(\alpha \rho+k \rho^{1+1 / n}\right) c^{2}
$$

with $k \leq 0$ in order to emphasize its analogy with the polytropic equation of state [33-36]. Indeed, it may be viewed as the sum of a linear equation of state $p=\alpha \rho c^{2}$ describing dust matter $(\alpha=0)$ or radiation $(\alpha=1 / 3)$ and a polytropic equation of state $p=k \rho^{1+1 / n} c^{2}$. We shall call (1) a generalized polytropic equation of state. At late times, this equation of state with $n<0$ leads to a constant energy density implying an exponential growth of the scale factor that is similar to the effect of the cosmological constant. At earlier times, and for $\alpha=0$, this equation of state returns the results of the dust matter model $(p \simeq 0)$. Therefore, this hydrodynamic model provides a unification of matter $\left(\rho \propto a^{-3}\right)$ and dark energy $\left(\rho=\rho_{\Lambda}\right)$ in the late universe [25-36].

In [34-36], we remarked that a constant pressure $p=$ $-\rho_{\Lambda} c^{2}$ has the same virtues as the Chaplygin gas model. (Actually, this is a particular case of the generalized Chaplygin gas model corresponding to $B=0$ and $a=0$.) This equation of state may be viewed as a generalized polytropic equation of state (1) with polytropic index $n=-1$, polytropic constant $k=-\rho_{\Lambda}$, and linear coefficient $\alpha=0$. At late times, this equation of state leads to a constant energy density equal to $\rho_{\Lambda}$ implying an exponential growth of the scale factor (late inflation). At earlier times, this equation of state returns the results of the dust matter model $(p \simeq 0)$. Therefore, as any Chaplygin gas model with $\alpha=0$ and $n<0$, this equation of state provides a unification of matter $\left(\rho \propto a^{-3}\right)$ and dark energy $\left(\rho=\rho_{\Lambda}\right)$ in the late universe. Moreover, this equation of state gives the same results as the standard $\Lambda \mathrm{CDM}$ model where matter and dark energy are treated as independent species. This is interesting since the $\Lambda \mathrm{CDM}$ model is consistent with observations and provides a good description of the late universe. (By contrast, the Chaplygin gas model is not consistent with observations unless $a$ is close to zero [37]. Therefore, observations tend to select the constant pressure equation of state $p=-\rho_{\Lambda} c^{2}(a=0, n=-1)$ among the whole family of Chaplygin gas models of the form $p=-A / \rho^{a}$.) In [34-36], we also proposed to describe the transition between the vacuum energy era and the radiation era in the early universe by a unique equation of state of the form $p / c^{2}=-4 \rho^{2} / 3 \rho_{P}+\rho / 3$. This equation of state may be viewed as a generalized polytropic equation of state (1) with polytropic index $n=1$, polytropic constant $k=-4 /\left(3 \rho_{P}\right)$, and linear coefficient $\alpha=1 / 3$. At early times, this equation of state leads to a constant energy density equal to $\rho_{P}$ implying an exponential growth of the scale factor (early inflation). At later times, and for $\alpha=1 / 3$, this equation of state returns the results of the radiation model $\left(p=\rho c^{2} / 3\right)$. Therefore, this hydrodynamic model provides a unification of vacuum energy $\left(\rho=\rho_{P}\right)$ and radiation $\left(\rho \propto a^{-4}\right)$ in the early universe. This equation of state may be viewed as an extension of the Chaplygin (or polytropic) gas model to the early universe. In this approach, the late universe is described by negative polytropic indices $n<0$ (e.g., $n=-1$ ) and the early universe by positive polytropic indices $n>0$ (e.g., $n=+1$ ) [34-36]. Indeed, for $n<0$, the polytropic equation of state dominates in the late universe where the density is low, and for $n>0$, the polytropic equation of state dominates in the early universe where the density is high.

In the viewpoint just exposed, one tries to "unify" vacuum energy + radiation on the one hand and dust matter + dark energy on the other hand. (It is oftentimes argued that the dark energy (cosmological constant) corresponds to the vacuum energy. This leads to the so-called cosmological problem [38] since the cosmological density $\rho_{\Lambda}=7.02$. $10^{-24} \mathrm{~g} / \mathrm{m}^{3}$ and the Planck density $\rho_{P}=5.16 \cdot 10^{99} \mathrm{~g} / \mathrm{m}^{3}$ differ by about 122 orders of magnitude. We think that it is a mistake to identify the dark energy with the vacuum energy. In this paper, we shall regard the vacuum energy and the dark energy as two distinct entities. We shall call vacuum energy the energy associated with the Planck density and dark energy the energy associated with the cosmological density. The vacuum energy is responsible for the inflation in the early universe and the dark energy for the inflation in the late universe. In this viewpoint, the vacuum energy is due to quantum mechanics and the dark energy is an effect of general relativity. The cosmological constant $\Lambda$ is interpreted as a fundamental constant of nature applying to the cosmophysics in the same way the Planck constant $\hbar$ applies to the microphysics.) In this paper, we adopt a different point of view. We propose that vacuum energy, radiation, and dark energy may be the manifestation of a unique form of "generalized radiation". We propose to describe it by a quadratic equation of state

$$
p=-\frac{4 \rho^{2}}{3 \rho_{P}} c^{2}+\frac{1}{3} \rho c^{2}-\frac{4}{3} \rho_{\Lambda} c^{2},
$$

involving the Planck density and the cosmological density. In the early universe, we recover the equation of state $p / c^{2}=$ $-4 \rho^{2} / 3 \rho_{P}+\rho / 3$ unifying vacuum energy and radiation. In 
the late universe, we obtain an equation of state $p / c^{2}=\rho / 3-$ $4 \rho_{\Lambda} / 3$ unifying radiation and dark energy. This equation of state may be viewed as a generalized polytropic equation of state (1) with polytropic index $n=-1$, polytropic constant $k=-4 \rho_{\Lambda} / 3$, and linear coefficient $\alpha=1 / 3$. The quadratic equation of state (2) "unifies" vacuum energy, radiation, and dark energy in a natural and simple manner. It also allows us to obtain a generalization of the Stefan-Boltzmann law for the evolution of the temperature [see (69)] that is valid during the whole evolution of the universe. In this approach, baryonic matter and dark matter are treated as different species. This is at variance with the usual scenario where vacuum energy + radiation on the one hand and dark matter + dark energy on the other hand are treated as different species.

The paper is organized as follows. In Section 2, we recall the basic equations of cosmology that will be needed in our study. In Section 3, we describe the transition between the vacuum energy era and the radiation era in the early universe. In Section 4, we describe the transition between the radiation era and the dark energy era in the late universe. In Section 5, we introduce the general model where vacuum energy + radiation + dark energy are described by a quadratic equation of state while baryonic matter and dark matter are considered as different species. We determine the general equations giving the evolution of the scale factor, density, and temperature (Section 5.1). We consider particular limits of these equations in the early universe (Section 5.2) and in the late universe (Sections 5.3 and 5.4). We connect these two limits and describe the whole evolution of the universe (Section 5.5). This model gives the same results as the standard $\Lambda \mathrm{CDM}$ model for $t>23.3 t_{P}$ and completes it by incorporating the early inflation in a natural manner [see (100)]. It also reveals a nice "symmetry" between the early universe (vacuum energy + radiation) and the late universe (radiation + dark energy). These two phases are described by polytropic equations of state with index $n=+1$ and $n=-1$, respectively. Furthermore, the cosmological density $\rho_{\Lambda}$ in the late universe plays a role similar to the Planck density $\rho_{P}$ in the early universe. They represent fundamental upper and lower density bounds differing by 122 orders of magnitude.

\section{Basic Equations of Cosmology}

In a space with uniform curvature, the line element is given by the Friedmann-Lemaître-Roberston-Walker (FLRW) metric

$$
d s^{2}=c^{2} d t^{2}-a(t)^{2}\left\{\frac{d r^{2}}{1-k r^{2}}+r^{2}\left(d \theta^{2}+\sin ^{2} \theta d \phi^{2}\right)\right\},
$$

where $a(t)$ represents the radius of curvature of the 3dimensional space, or the scale factor. (In our conventions, $r$ and $k$ are dimensionless, while the scale factor $a(t)$ has the dimension of a length. It is defined such that $a_{0}=c / H_{0}$ (where $c$ is the speed of light and $H_{0}$ is the Hubble constant) at the present epoch. This corresponds to the cosmological horizon. The usual (dimensionless) scale factor is $\widetilde{a}(t)=$ $a(t) / a_{0}$. It satisfies $\tilde{a}_{0}=1$ at the present epoch.) By an abuse of language, we shall sometimes call it the "radius of the universe." On the other hand, $k$ determines the curvature of space. The universe may be closed $(k>0)$, flat $(k=0)$, or open $(k<0)$.

If the universe is isotropic and homogeneous at all points in conformity with the line element (3) and contains a uniform perfect fluid of energy density $\epsilon(t)=\rho(t) c^{2}$ and isotropic pressure $p(t)$, the energy-momentum tensor $T_{j}^{i}$ is

$$
T_{0}^{0}=\rho c^{2}, \quad T_{1}^{1}=T_{2}^{2}=T_{3}^{3}=-p .
$$

The Einstein equations

$$
R_{j}^{i}-\frac{1}{2} g_{j}^{i} R-\Lambda g_{j}^{i}=-\frac{8 \pi G}{c^{2}} T_{j}^{i}
$$

relate the geometrical structure of the spacetime $\left(g_{i j}\right)$ to the material content of the universe $\left(T_{i j}\right)$. For the sake of generality, we have accounted for a possibly nonzero cosmological constant $\Lambda$. Given Equations (3) and (4), these equations reduce to

$$
\begin{gathered}
8 \pi G \rho+\Lambda=3 \frac{\dot{a}^{2}+k c^{2}}{a^{2}}, \\
\frac{8 \pi G}{c^{2}} p-\Lambda=-\frac{2 a \ddot{a}+\dot{a}^{2}+k c^{2}}{a^{2}},
\end{gathered}
$$

where dots denote differentiation with respect to time. These are the well-known cosmological equations describing a nonstatic universe first derived by Friedmann [6].

The Friedmann equations are usually written in the form

$$
\begin{gathered}
\frac{d \rho}{d t}+3 \frac{\dot{a}}{a}\left(\rho+\frac{p}{c^{2}}\right)=0, \\
\frac{\ddot{a}}{a}=-\frac{4 \pi G}{3}\left(\rho+\frac{3 p}{c^{2}}\right)+\frac{\Lambda}{3}, \\
H^{2}=\left(\frac{\dot{a}}{a}\right)^{2}=\frac{8 \pi G}{3} \rho-\frac{k c^{2}}{a^{2}}+\frac{\Lambda}{3},
\end{gathered}
$$

where we have introduced the Hubble parameter $H=\dot{a} / a$. Among these three equations, only two are independent. The first equation, which can be viewed as an "equation of continuity", can be directly derived from the conservation of the energy momentum tensor $\partial_{i} T^{i j}=0$, which results from the Bianchi identities. For a given barotropic equation of state $p=p(\rho)$, it determines the relation between the density and the scale factor. Then, the temporal evolution of the scale factor is given by (9).

Equivalent expressions of the "equation of continuity" are

$$
\begin{gathered}
a \frac{d \rho}{d a}=-3\left(\rho+\frac{p}{c^{2}}\right), \\
\frac{d}{d a}\left(\rho a^{3}\right)=-3 \frac{p}{c^{2}} a^{2}, \\
a^{3} \frac{d p}{d t}=\frac{d}{d t}\left[a^{3}\left(p+\rho c^{2}\right)\right] .
\end{gathered}
$$

Introducing the volume $V=(4 / 3) \pi a^{3}$ and the energy $E=$ $\rho c^{2} V$, (11) becomes $d E=-p d V$. This can be viewed as the 
first principle of thermodynamics for an adiabatic evolution of the universe $d S=0$.

From the first principle of thermodynamics in the form $d S(V, T)=(1 / T)\left[d\left(\rho(T) c^{2} V\right)+p(T) d V\right]$, one can derive the thermodynamical equation [6]

$$
\frac{d p}{d T}=\frac{1}{T}\left(\rho c^{2}+p\right)
$$

For a given barotropic equation of state $p=p(\rho)$, this equation can be integrated to obtain the relation $T=T(\rho)$ between the temperature and the density. Combining (12) with (13), we get [6] that

$$
\dot{S}=0, \quad \text { where } S=\frac{a^{3}}{T}\left(p+\rho c^{2}\right)
$$

is the entropy of the universe in a volume $a^{3}$. This confirms that the Friedmann equations (7)-(9) imply the conservation of the entropy.

In this paper, we consider a flat universe $(k=0)$ in agreement with the observations of the cosmic microwave background (CMB) [1]. On the other hand, we set $\Lambda=0$ because the effect of the cosmological constant will be taken into account in the equation of state. The Friedmann equations then reduce to

$$
\begin{aligned}
& \frac{d \rho}{d t}+3 \frac{\dot{a}}{a}\left(\rho+\frac{p}{c^{2}}\right)=0, \\
& \frac{\ddot{a}}{a}=-\frac{4 \pi G}{3}\left(\rho+\frac{3 p}{c^{2}}\right), \\
& H^{2}=\left(\frac{\dot{a}}{a}\right)^{2}=\frac{8 \pi G}{3} \rho .
\end{aligned}
$$

The deceleration parameter is defined by

$$
q(t)=-\frac{\ddot{a} a}{\dot{a}^{2}} .
$$

The universe is decelerating when $q>0$ and accelerating when $q<0$. Introducing the equation of state parameter $w=p / \rho c^{2}$ and using the Friedmann equations (16) and (17), we obtain for a flat universe

$$
q(t)=\frac{1+3 w(t)}{2} .
$$

We see from (19) that the universe is decelerating if $w>-1 / 3$ (strong energy condition) and accelerating if $w<-1 / 3$. On the other hand, according to (15), the density decreases with the scale factor if $w>-1$ (null dominant energy condition) and increases with the scale factor if $w<-1$.

\section{The Early Universe}

In [34-36], we have proposed to describe the transition between the vacuum energy era and the radiation era in the early universe by a single equation of state of the form

$$
p=\frac{1}{3} \rho c^{2}-\frac{4 \rho^{2}}{3 \rho_{P}} c^{2}
$$

where $\rho_{P}=c^{5} / G^{2} \hbar=5.16 \cdot 10^{99} \mathrm{~g} / \mathrm{m}^{3}$ is the Planck density. This equation of state corresponds to a generalized polytropic equation of state (1) with $\alpha=1 / 3, n=1$, and $k=-4 /\left(3 \rho_{P}\right)$. For $\rho \ll \rho_{P}$, we recover the equation of state of the pure radiation $p \sim \rho c^{2} / 3$. For $\rho \rightarrow \rho_{P}$, we get $p \rightarrow-\rho_{P} c^{2}$ corresponding to the vacuum energy.

The continuity equation (15) may be integrated into

$$
\rho=\frac{\rho_{P}}{\left(a / a_{1}\right)^{4}+1},
$$

where $a_{1}$ is a constant of integration. This characteristic scale marks the transition between the vacuum energy era and the radiation era. The equation of state (20) interpolates smoothly between the vacuum energy era $\left(p=-\rho c^{2}\right.$ and $\left.\rho=\rho_{P}\right)$ and the radiation era $\left(p=\rho c^{2} / 3\right.$ and $\left.\rho_{\mathrm{rad}} \sim \rho_{P} a_{1}^{4} / a^{4}\right)$. It provides therefore a "unified" description of vacuum energy and radiation in the early universe. This amounts to summing the inverse of the densities of these two phases. Indeed, (21) may be rewritten as

$$
\frac{1}{\rho}=\frac{1}{\rho_{\mathrm{rad}}}+\frac{1}{\rho_{P}} .
$$

At $a=a_{1}$ we have $\rho_{\text {rad }}=\rho_{P}$ so that $\rho=\rho_{P} / 2$.

The equation of state parameter $w=p / \rho c^{2}$ and the deceleration parameter $q$ are given by

$$
w=\frac{1}{3}-\frac{4 \rho}{3 \rho_{P}}, \quad q=1-2 \frac{\rho}{\rho_{P}} .
$$

The velocity of sound $c_{s}^{2}=p^{\prime}(\rho)$ is given by

$$
\frac{c_{s}^{2}}{c^{2}}=\frac{1}{3}-\frac{8}{3} \frac{\rho}{\rho_{P}} .
$$

As the universe expands from $a=0$ to $a=+\infty$, the density decreases from $\rho_{P}$ to 0 , the equation of state parameter $w$ increases from -1 to $1 / 3$, the deceleration parameter $q$ increases from -1 to 1 , and the ratio $\left(c_{s} / c\right)^{2}$ increases from $-7 / 3$ to $1 / 3$.

3.1. The Vacuum Energy Era: Early Inflation. When $a \ll a_{1}$, the density tends to a maximum value

$$
\rho=\rho_{\max }=\rho_{P}
$$

and the pressure tends to $p=-\rho_{P} c^{2}$. The Planck density $\rho_{P}=$ $5.16 \cdot 10^{99} \mathrm{~g} / \mathrm{m}^{3}$ (vacuum energy) represents a fundamental upper bound for the density. A constant value of the density $\rho \simeq \rho_{P}$ gives rise to a phase of early inflation. From the Friedmann equation (17), we find that the Hubble parameter is constant $H=(8 \pi / 3)^{1 / 2} t_{P}^{-1}$ where we have introduced the Planck time $t_{P}=1 /\left(G \rho_{P}\right)^{1 / 2}=\left(\hbar G / c^{5}\right)^{1 / 2}$. Numerically, $H=$ $5.37 \cdot 10^{43} \mathrm{~s}^{-1}$. Therefore, the scale factor increases exponentially rapidly with time as

$$
a(t) \sim l_{P} e^{(8 \pi / 3)^{1 / 2} t / t_{P}}
$$


The timescale of the exponential growth is the Planck time $t_{P}=5.39 \cdot 10^{-44} \mathrm{~s}$. We have defined the "original" time $t=0$ such that $a(0)$ is equal to the Planck length $l_{P}=c t_{P}=$ $\left(G \hbar / c^{3}\right)^{1 / 2}=1.62 \cdot 10^{-35} \mathrm{~m}$. Mathematically speaking, the universe exists at any time in the past $\left(a \rightarrow 0\right.$ and $\rho \rightarrow \rho_{P}$ for $t \rightarrow-\infty)$, so there is no primordial singularity. However, when $a \rightarrow 0$, we cannot ignore quantum fluctuations associated with the spacetime. In that case, we cannot use the classical Einstein equations anymore and a theory of quantum gravity is required. It is not known whether quantum gravity will remove, or not, the primordial singularity. Therefore, we cannot extrapolate the solution (26) to the infinite past. However, this solution may provide a semiclassical description of the phase of early inflation when $a>l_{P}$.

3.2. The Radiation Era. When $a \gg a_{1}$, we recover the equation

$$
\rho_{\text {rad }} \sim \frac{\rho_{P} a_{1}^{4}}{a^{4}}
$$

corresponding to the pure radiation described by an equation of state $p=\rho c^{2} / 3$. The conservation of $\rho_{\text {rad }} a^{4}$ implies that $\rho_{P} a_{1}^{4}=\rho_{\text {rad }, 0} a_{0}^{4}$ where $\rho_{\text {rad }, 0}$ is the present density of radiation and $a_{0}=c / H_{0}=1.32 \cdot 10^{26} \mathrm{~m}$ the present distance of cosmological horizon determined by the Hubble constant $H_{0}=2.27 \cdot 10^{-18} \mathrm{~s}^{-1}$ (the Hubble time is $H_{0}^{-1}=4.41 \cdot 10^{17} \mathrm{~s}$ ). Writing $\rho_{\mathrm{rad}, 0}=\Omega_{\mathrm{rad}, 0} \rho_{0}$ where $\rho_{0}=3 H_{0}^{2} / 8 \pi G=9.20$. $10^{-24} \mathrm{~g} / \mathrm{m}^{3}$ is the present density of the universe given by (17) and $\Omega_{\mathrm{rad}, 0}=8.48 \cdot 10^{-5}$ is the present fraction of radiation in the universe [1], we obtain

$$
a_{1}=2.61 \cdot 10^{-6} \mathrm{~m} \text {. }
$$

This scale is intermediate between the Planck length $l_{P}$ and the present size of the universe $a_{0}\left(a_{1} / l_{P}=1.61 \cdot 10^{29}\right.$ and $\left.a_{1} / a_{0}=1.97 \cdot 10^{-32}\right)$. It gives the typical size of the universe at the end of the inflationary phase (or at the beginning of the radiation era). When $a \gg a_{1}$, the Friedmann equation (17) yields

$$
\frac{a}{a_{1}} \sim\left(\frac{32 \pi}{3}\right)^{1 / 4}\left(\frac{t}{t_{P}}\right)^{1 / 2}
$$

We then have

$$
\frac{\rho}{\rho_{P}} \sim \frac{3}{32 \pi}\left(\frac{t_{P}}{t}\right)^{2} .
$$

During the radiation era, the density decreases algebraically as the universe expands.

3.3. The General Solution. The general solution of the Friedmann equation (17) with (21) is

$$
\begin{gathered}
\sqrt{\left(a / a_{1}\right)^{4}+1}-\ln \left(\frac{1+\sqrt{\left(a / a_{1}\right)^{4}+1}}{\left(a / a_{1}\right)^{2}}\right) \\
=2\left(\frac{8 \pi}{3}\right)^{1 / 2} \frac{t}{t_{P}}+C,
\end{gathered}
$$

where $C$ is a constant of integration. It is determined such that $a=l_{P}$ at $t=0$. Setting $\epsilon=l_{P} / a_{1}$, we get

$$
C(\epsilon)=\sqrt{\epsilon^{4}+1}-\ln \left(\frac{1+\sqrt{\epsilon^{4}+1}}{\epsilon^{2}}\right) .
$$

Numerically, $\epsilon=6.20 \cdot 10^{-30}$ and $C=-134$. For $t \rightarrow-\infty$, we have the exact asymptotic result

$$
a(t) \sim a_{1} e^{(8 \pi / 3)^{1 / 2} t / t_{P}+D},
$$

with $D=(C+\ln 2-1) / 2$. Due to the smallness of $\epsilon$, a very good approximation of $C$ and $D$ is given by $C \simeq 1-\ln 2+2 \ln \epsilon$ and $D \simeq \ln \epsilon$. With this approximation, (33) returns (26). Using (23), the universe is accelerating when $a<a_{c}$ (i.e., $\rho>\rho_{c}$ ) and decelerating when $a>a_{c}$ (i.e., $\rho<\rho_{c}$ ) where $a_{c}=a_{1}$ and $\rho_{c}=\rho_{P} / 2$. The time $t_{c}$ at which the universe starts decelerating is given by $t_{c}=(3 / 32 \pi)^{1 / 2}[\sqrt{2}-\ln (1+\sqrt{2})-C] t_{P}$. This corresponds to the time at which the curve $a(t)$ presents an inflexion point. It turns out that this inflexion point $a_{c}$ coincides with $a_{1}$ so it also marks the end of the inflation $\left(t_{c}=t_{1}\right)$. Numerically, $\rho_{c}=\rho_{1}=0.5 \rho_{P}=2.58 \cdot 10^{99} \mathrm{~g} / \mathrm{m}^{3}$, $a_{c}=a_{1}=2.61 \cdot 10^{-6} \mathrm{~m}$, and $t_{c}=t_{1}=23.3 t_{P}=1.25 \cdot 10^{-42} \mathrm{~s}$. At $t=t_{i}=0, a_{i}=l_{P}$ and $\rho_{i} \simeq \rho_{P}$. Numerically, $a_{i}=l_{P}=$ $1.62 \cdot 10^{-35} \mathrm{~m}$ and $\rho_{i} \simeq \rho_{P}=5.16 \cdot 10^{99} \mathrm{~g} / \mathrm{m}^{3}$.

3.4. The Temperature and the Pressure. The thermodynamical equation (13) with the equation of state (20) may be integrated into

$$
T=T_{P}\left(\frac{15}{\pi^{2}}\right)^{1 / 4}\left(1-\frac{\rho}{\rho_{P}}\right)^{7 / 4}\left(\frac{\rho}{\rho_{P}}\right)^{1 / 4}
$$

where $T_{P}$ is a constant of integration. Using (21), we get

$$
T=T_{P}\left(\frac{15}{\pi^{2}}\right)^{1 / 4} \frac{\left(a / a_{1}\right)^{7}}{\left[\left(a / a_{1}\right)^{4}+1\right]^{2}} .
$$

Equation (34) may be seen as a generalized Stefan-Boltzmann law in the early universe. In the radiation era $\rho \ll \rho_{P}$, it reduces to the ordinary Stefan-Boltzmann law

$$
T=T_{P}\left(\frac{15}{\pi^{2}}\right)^{1 / 4}\left(\frac{\rho}{\rho_{P}}\right)^{1 / 4} .
$$

This allows us to identify the constant of integration $T_{P}$ with the Planck temperature $T_{P}=\left(\hbar c^{5} / G k_{B}^{2}\right)^{1 / 2}=1.42 \cdot 10^{32} \mathrm{~K}$. On the other hand, in the inflationary phase $\rho \rightarrow \rho_{P}$, the temperature is related to the density by

$$
\frac{T}{T_{P}} \simeq\left(\frac{15}{\pi^{2}}\right)^{1 / 4}\left(1-\frac{\rho}{\rho_{P}}\right)^{7 / 4} .
$$

According to (35), $T \sim T_{P}\left(15 / \pi^{2}\right)^{1 / 4}\left(a / a_{1}\right)^{7}$ for $a \ll a_{1}$ and $T \sim T_{P}\left(15 / \pi^{2}\right)^{1 / 4}\left(a_{1} / a\right)$ for $a \gg a_{1}$.

The temperature starts from $T=0$ at $t=-\infty$, increases exponentially rapidly during the inflation as

$$
\frac{T}{T_{P}} \sim\left(\frac{15}{\pi^{2}}\right)^{1 / 4}\left(\frac{l_{P}}{a_{1}}\right)^{7} e^{7(8 \pi / 3)^{1 / 2} t / t_{P}}
$$


reaches a maximum value $T_{e}$ and decreases algebraically as

$$
\frac{T}{T_{P}} \sim\left(\frac{45}{32 \pi^{3}}\right)^{1 / 4}\left(\frac{t_{P}}{t}\right)^{1 / 2},
$$

during the radiation era. Using (34) and (35), we find that the point corresponding to the maximum temperature is $\rho_{e}=$ $\rho_{P} / 8, a_{e}=7^{1 / 4} a_{1}, T_{e}=(7 / 8)^{7 / 4}\left(15 / 8 \pi^{2}\right)^{1 / 4} T_{P}$. It is reached at a time $t_{e}=(3 / 32 \pi)^{1 / 2}[\sqrt{8}-\ln (1+\sqrt{8})+\ln (7) / 2-C] t_{P}$. Numerically, $\rho_{e}=0.125 \rho_{P}=6.44 \cdot 10^{98} \mathrm{~g} / \mathrm{m}^{3}, a_{e}=1.63 a_{1}=$ $4.24 \cdot 10^{-6} \mathrm{~m}, T_{e}=0.523 T_{P}=7.40 \cdot 10^{31} \mathrm{~K}$, and $t_{e}=23.6 t_{P}=$ $1.27 \cdot 10^{-42} \mathrm{~s}$. At $t=t_{i}=0, T_{i} \simeq T_{P}\left(15 / \pi^{2}\right)^{1 / 4}\left(l_{P} / a_{1}\right)^{7}$. Numerically, $T_{i}=3.91 \cdot 10^{-205} T_{P}=5.54 \cdot 10^{-173} \mathrm{~K}$. At $t=t_{c}=t_{1}, T_{c}=(1 / 4)\left(15 / \pi^{2}\right)^{1 / 4} T_{P}$. Numerically, $T_{c}=T_{1}=$ $0.278 T_{P}=3.93 \cdot 10^{31} \mathrm{~K}$.

The pressure is given by (20). Using (21), we get

$$
p=\frac{1}{3} \frac{\left(a / a_{1}\right)^{4}-3}{\left[\left(a / a_{1}\right)^{4}+1\right]^{2}} \rho_{P} c^{2} .
$$

The pressure starts from $p=-\rho_{P} c^{2}$ at $t=-\infty$, remains approximately constant during the inflation, becomes positive, reaches a maximum value $p_{e}$, and decreases algebraically during the radiation era. At $t=t_{i}=0, p_{i} \simeq-\rho_{P} c^{2}$. Numerically, $p_{i}=-4.64 \cdot 10^{116} \mathrm{~g} / \mathrm{m} \mathrm{s}^{2}$. The point at which the pressure vanishes is $a_{w}=3^{1 / 4} a_{1}, \rho_{w}=\rho_{P} / 4, T_{w}=(9 / 16)\left(5 / \pi^{2}\right)^{1 / 4} T_{P}$. This corresponds to a time $t_{w}=(3 / 32 \pi)^{1 / 2}[2-(1 / 2) \ln (3)-$ $C] t_{P}$. Numerically, $a_{w}=1.32 a_{1}=3.43 \cdot 10^{-6} \mathrm{~m}, \rho_{w}=0.25 \rho_{P}=$ $1.29 \cdot 10^{99} \mathrm{~g} / \mathrm{m}^{3}, T_{w}=0.475 T_{P}=6.74 \cdot 10^{31} \mathrm{~K}$, and $t_{w}=$ $23.4 t_{P}=1.26 \cdot 10^{-42} \mathrm{~s}$. On the other hand, the pressure reaches its maximum $p_{e}=\rho_{P} c^{2} / 48$ at the same point as the one at which the temperature reaches its maximum. Numerically, $p_{e}=9.66 \cdot 10^{114} \mathrm{~g} / \mathrm{m} \mathrm{s}^{2}$. At $t=t_{c}=t_{1}$, we have $p_{c}=p_{1}=$ $-\rho_{P} c^{2} / 6$. Numerically, $p_{c}=p_{1}=-7.73 \cdot 10^{115} \mathrm{~g} / \mathrm{m} \mathrm{s}^{2}$.

Using (20), (21), and (35), we find that the entropy (14) is given by

$$
S=\frac{4}{3}\left(\frac{\pi^{2}}{15}\right)^{1 / 4} \frac{\rho_{P} c^{2}}{T_{P}} a_{1}^{3},
$$

and we check that it is a constant. Numerically, $S / k_{B}=5.04$. $10^{87}$.

3.5. The Evolution of the Early Universe. In our model, the universe starts at $t=-\infty$ with a vanishing radius $a=0$, a finite density $\rho=\rho_{P}=5.16 \cdot 10^{99} \mathrm{~g} / \mathrm{m}^{3}$, a finite pressure $p=-\rho_{P} c^{2}=-4.64 \cdot 10^{116} \mathrm{~g} / \mathrm{m} \mathrm{s}^{2}$, and a vanishing temperature $T=0$. The universe exists at any time in the past and does not present any singularity. For $t<0$, the radius of the universe is less than the Planck length $l_{P}=1.62 \cdot 10^{-35} \mathrm{~m}$. In the Planck era, quantum gravity should be taken into account and our semiclassical approach is probably not valid in the infinite past. At $t=t_{i}=0$, the radius of the universe is equal to the Planck length $a_{i}=l_{P}=1.62 \cdot 10^{-35} \mathrm{~m}$. The corresponding density, temperature, and pressure are $\rho_{i} \simeq$ $\rho_{P}=5.16 \cdot 10^{99} \mathrm{~g} / \mathrm{m}^{3}, T_{i}=3.91 \cdot 10^{-205} T_{P}=5.54 \cdot 10^{-173} \mathrm{~K}$, and $p_{i} \simeq-\rho_{P} c^{2}=-4.64 \cdot 10^{116} \mathrm{~g} / \mathrm{m} \mathrm{s}^{2}$. We note that quantum mechanics regularizes the finite time singularity present in the standard Big Bang theory. This is similar to finite size effects in second-order phase transitions (see Section 3.6). The Big Bang theory is recovered for $\hbar=0$. We also note that the universe is very cold at $t=t_{i}=0$, contrary to what is predicted by the Big Bang theory (a naive extrapolation of the law $T \propto t^{-1 / 2}$ leads to $\left.T(0)=+\infty\right)$. The universe first undergoes a phase of inflation during which its radius and temperature increase exponentially rapidly, while its density and pressure remain approximately constant. The inflation "starts" at $t_{i}=0$ and ends at $t_{1}=23.3 t_{P}=1.25 \cdot 10^{-42} \mathrm{~s}$. During this very short lapse of time, the radius of the universe grows from $a_{i}=l_{P}=$ $1.62 \cdot 10^{-35} \mathrm{~m}$ to $a_{1}=2.61 \cdot 10^{-6} \mathrm{~m}$, and the temperature grows from $T_{i}=3.91 \cdot 10^{-205} T_{P}=5.54 \cdot 10^{-173} \mathrm{~K}$ to $T_{1}=0.278 T_{P}=$ $3.93 \cdot 10^{31} \mathrm{~K}$. By contrast, the density and the pressure do not change significatively: they go from $\rho_{i} \simeq \rho_{P}=5.16 \cdot 10^{99} \mathrm{~g} / \mathrm{m}^{3}$ and $p_{i} \simeq-\rho_{P} c^{2}=-4.64 \cdot 10^{116} \mathrm{~g} / \mathrm{m} \mathrm{s}^{2}$ to $\rho_{1}=0.5 \rho_{P}=$ $2.58 \cdot 10^{99} \mathrm{~g} / \mathrm{m}^{3}$ and $p_{1}=-0.167 \rho_{P} c^{2}=-7.73 \cdot 10^{115} \mathrm{~g} / \mathrm{m} \mathrm{s}^{2}$. The pressure passes from negative values to positive values at $t_{w}=23.4 t_{P}=1.26 \cdot 10^{-42} \mathrm{~s}$ corresponding to $\rho_{w}=$ $0.25 \rho_{P}=1.29 \cdot 10^{99} \mathrm{~g} / \mathrm{m}^{3}, a_{w}=1.32 a_{1}=3.43 \cdot 10^{-6} \mathrm{~m}$, and $T_{w}=0.475 T_{P}=6.74 \cdot 10^{31} \mathrm{~K}$. After the inflation, the universe enters in the radiation era and, from that point, we recover the standard model [6]. The radius increases algebraically as $a \propto t^{1 / 2}$, while the density and the temperature decrease algebraically as $\rho \propto t^{-2}$ and $T \propto t^{-1 / 2}$. The temperature and the pressure achieve their maximum values $T_{e}=0.523 T_{P}=$ $7.40 \cdot 10^{31} \mathrm{~K}$ and $p_{e}=2.08 \cdot 10^{-2} \rho_{P} c^{2}=9.66 \cdot 10^{114} \mathrm{~g} / \mathrm{m} \mathrm{s}^{2}$ at $t=t_{e}=23.6 t_{P}=1.27 \cdot 10^{-42} \mathrm{~s}$. At that moment, the density is $\rho_{e}=0.125 \rho_{P}=6.44 \cdot 10^{98} \mathrm{~g} / \mathrm{m}^{3}$ and the radius $a_{e}=1.63 a_{1}=4.24 \cdot 10^{-6} \mathrm{~m}$. During the inflation, the universe is accelerating and during the radiation era it is decelerating. The transition (marked by an inflexion point) takes place at a time $t_{c}=t_{1}=23.3 t_{P}=1.25 \cdot 10^{-42} \mathrm{~s}$ coinciding with the end of the inflation $\left(a_{c}=a_{1}\right)$. The evolution of the scale factor, density, and temperature as a function of time are represented in Figures 1, 2, 3, 4, 5, 6, and 7 in logarithmic and linear scales.

We note that the inflationary process described previously is relatively different from the usual inflationary scenario [2-5]. In standard inflation, the universe is radiation dominated up to $t_{i}=10^{-35} \mathrm{~s}$ but expands exponentially by a factor $10^{30}$ in the interval $t_{i}<t<t_{f}$ with $t_{f}=10^{-33} \mathrm{~s}$. For $t>$ $t_{f}$, the evolution is again radiation dominated. At $t=t_{i}$, the temperature is about $10^{27} \mathrm{~K}$ (this corresponds to the epoch at which most "grand unified theories" have a significant influence on the evolution of the universe). During the exponential inflation, the temperature drops drastically; however, the matter is expected to be reheated to the initial temperature of $10^{27} \mathrm{~K}$ by various high energy processes [39]. In the inflationary process described previously, the evolution of the temperature, given by a generalized Stefan-Boltzmann law, is different. It is initially very low and increases during the inflation.

3.6. Analogy with Phase Transitions. The standard Big Bang theory is a classical theory in which quantum effects are neglected. In that case, it exhibits a finite time singularity: 


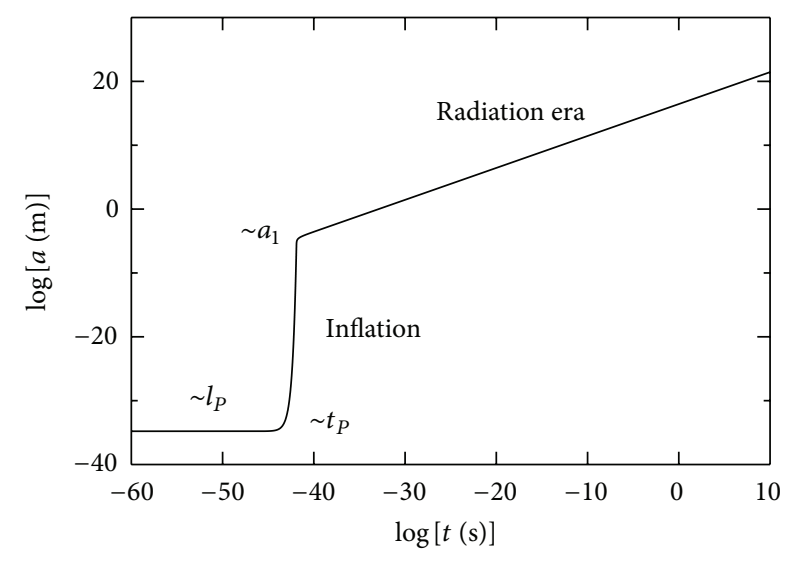

FIgURE 1: Evolution of the scale factor $a$ with the time $t$ in logarithmic scales. This figure clearly shows the phase of inflation connecting the vacuum energy era to the radiation era. During the inflation, the scale factor increases by 29 orders of magnitude in less than $10^{-42} \mathrm{~s}$. In the radiation era, $a$ increases algebraically.

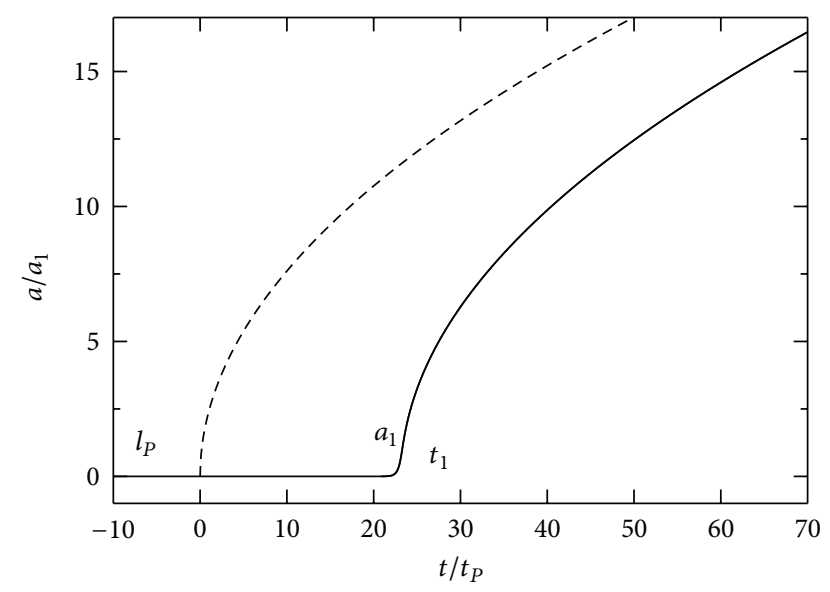

FIgURE 2: Evolution of the scale factor $a$ with the time $t$ in linear scales. The dashed line corresponds to the standard radiation model of Section 3.2 exhibiting a finite time singularity at $t=0$ (Big Bang). When quantum mechanics is taken into account (as in our simple semiclassical model), the initial singularity is smoothed-out and the scale factor at $t=0$ is equal to the Planck length $l_{P}=1.62 \cdot 10^{-35} \mathrm{~m}$. This is similar to a second order phase transition where the Planck constant plays the role of finite size effects (see Section 3.6 for a development of this analogy).

the radius of the universe is equal to zero at $t=0$, while its density and its temperature are infinite. For $t<0$, the solution is not defined and we may take $a=0$. For $t>0$, the radius of the universe increases as $a \propto t^{1 / 2}$. This is similar to a second-order phase transition if we view the time $t$ as the control parameter (e.g., the temperature $T$ ) and the scale factor $a$ as the order parameter (e.g., the magnetization $M$ ). The exponent $1 / 2$ is the same as in mean field theories of second-order phase transitions (i.e., $\left.M \sim\left(T_{c}-T\right)^{1 / 2}\right)$, but this is essentially a coincidence.

When quantum mechanics effects are taken into account, as in our simple semiclassical model, the singularity at $t=0$

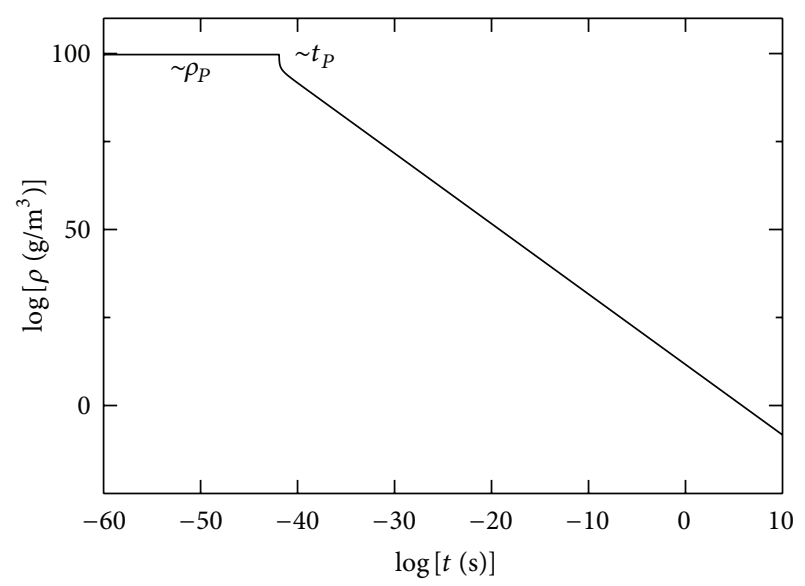

Figure 3: Evolution of the density $\rho$ with the time $t$ in logarithmic scales. During the inflation, the density remains approximately constant with the Planck value $\rho_{\max }=\rho_{P}$ which represents an upper bound. In the radiation era, $\rho$ decreases algebraically.

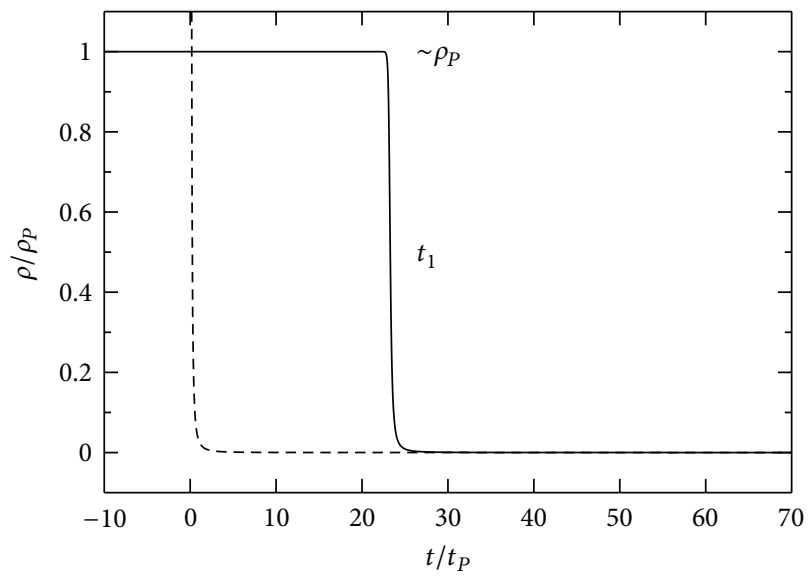

FIGURE 4: Evolution of the density $\rho$ with the time $t$ in linear scales. The dashed line corresponds to the standard radiation model of Section 3.2 exhibiting a finite time singularity at $t=0$. Quantum mechanics limits the rise of the density to the Planck value $\rho_{P}=$ $5.16 \cdot 10^{99} \mathrm{~g} / \mathrm{m}^{3}$.

disappears and the curves $a(t), \rho(t)$ and $T(t)$ are regularized. In particular, we find that $a=l_{P}>0$ at $t=0$, instead of $a=0$, due to the finite value of $\hbar$. This is similar to the regularization due to finite size effects (e.g., the system size $L$ or the number of particles $N$ ) in ordinary phase transitions. In this sense, the classical limit $\hbar \rightarrow 0$ is similar to the thermodynamic limit $(L \rightarrow+\infty$ or $N \rightarrow+\infty)$ in ordinary phase transitions.

To study the convergence toward the classical Big Bang solution when $\hbar \rightarrow 0$, it is convenient to use a proper normalization of the parameters. We call $\hbar_{0}=1.05 \cdot 10^{-34} \mathrm{~J} \mathrm{~s}$ the true value of the Planck constant (in this section, the subscript 0 refers to true values of the parameters). Then, we express time in terms of $t_{P}^{0}$ and lengths in terms of $a_{1}^{0}$. 


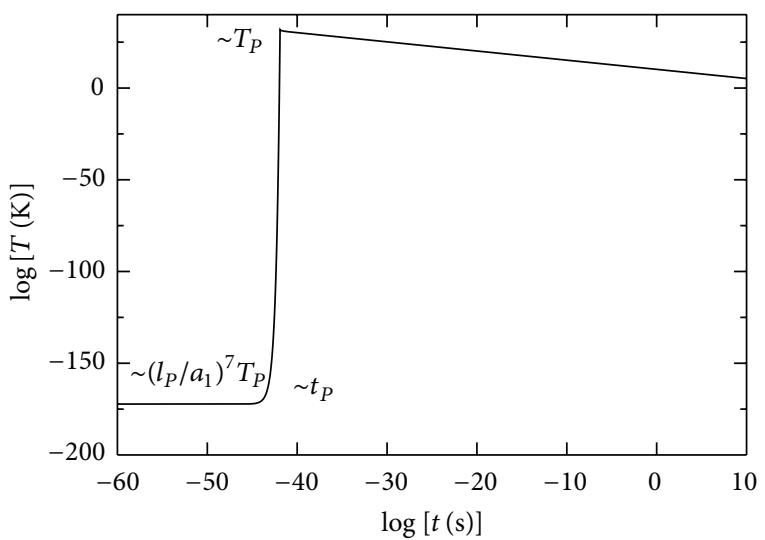

FIgURE 5: Evolution of the temperature $T$ with the time $t$ in logarithmic scales. For $t \leq 0$, the universe is extremely cold $(T<$ $10^{-173} \mathrm{~K}$ ). During the inflation, the temperature increases by 204 orders of magnitude in less than $10^{-42} \mathrm{~s}$. During the radiation era, the temperature decreases algebraically.

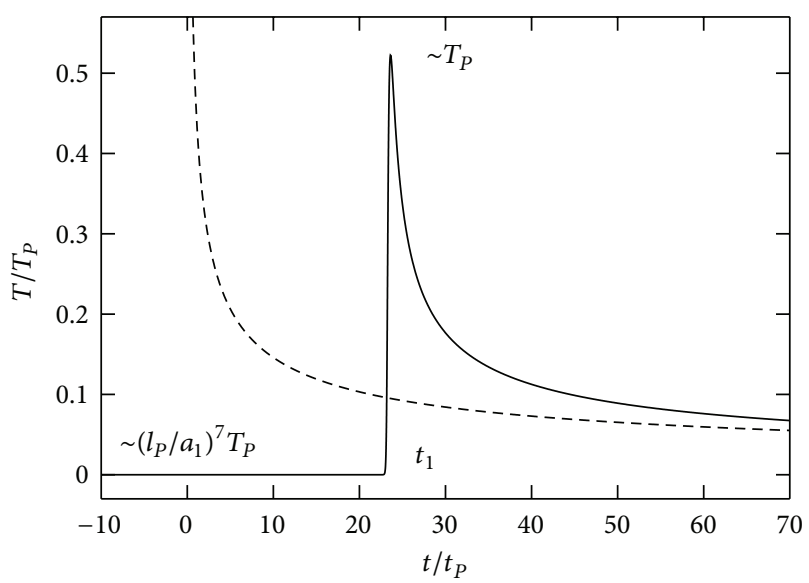

FIGURE 6: Evolution of the temperature $T$ with the time $t$ in linear scales. The dashed line corresponds to the standard radiation model of Section 3.2 exhibiting a finite time singularity at $t=0$. Quantum mechanics limits the rise of the temperature to about half the Planck value $T_{P}=1.42 \cdot 10^{32} \mathrm{~K}$. In the vacuum energy era, the temperature drops to zero exponentially rapidly for $t \rightarrow-\infty$.

We introduce $\widehat{a}=a / a_{1}^{0}$ and $\widehat{t}=t / t_{P}^{0}$. Using $l_{P}=c t_{P}$ and $\rho_{P} a_{1}^{4}=\rho_{\text {rad }, 0} a_{0}^{4}$, we obtain

$$
\frac{l_{P}}{l_{P}^{0}}=\frac{t_{P}}{t_{P}^{0}}=\left(\frac{a_{1}}{a_{1}^{0}}\right)^{2}=\widehat{\hbar}^{1 / 2}
$$

where $\widehat{\hbar}=\hbar / \hbar_{0}$. Therefore

$$
\frac{\epsilon}{\epsilon_{0}}=\frac{l_{P} / a_{1}}{l_{P}^{0} / a_{1}^{0}}=\frac{a_{1}}{a_{1}^{0}}=\widehat{\hbar}^{1 / 4}
$$

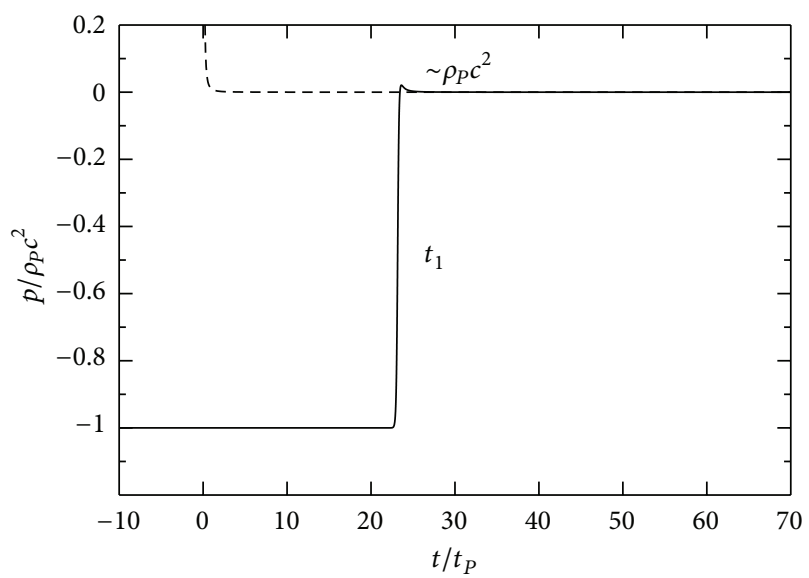

FIGURE 7: Evolution of the pressure $p$ with the time $t$ in linear scales. The dashed line corresponds to the standard radiation model of Section 3.2 exhibiting a finite time singularity at $t=0$. Quantum mechanics limits the rise of the pressure to $\rho_{P} c^{2} / 48=9.66$. $10^{114} \mathrm{~g} / \mathrm{m} \mathrm{s}^{2}$. In the vacuum energy era, the pressure decreases exponentially rapidly, becomes negative, and tends to $-\rho_{P} c^{2}=$ $-4.64 \cdot 10^{116} \mathrm{~g} / \mathrm{m} \mathrm{s}^{2}$ for $t \rightarrow-\infty$.

where $\epsilon_{0}=l_{P}^{0} / a_{1}^{0}=6.20 \cdot 10^{-30}$. Using these relations and (31), we get

$$
\begin{aligned}
& \sqrt{\frac{\widehat{a}^{4}}{\widehat{\hbar}}+1}-\ln \left(\frac{1+\sqrt{\widehat{a}^{4} / \widehat{\hbar}+1}}{\widehat{a}^{2} / \widehat{\hbar}^{1 / 2}}\right) \\
& =2\left(\frac{8 \pi}{3}\right)^{1 / 2} \frac{\widehat{t}}{\widehat{\hbar}^{1 / 2}}+C\left(\epsilon_{0} \widehat{\hbar}^{1 / 4}\right),
\end{aligned}
$$

where $C(\epsilon)$ is defined by (32). This equation describes a phase transition between the vacuum energy era and the radiation era. The normalized Planck constant $\widehat{\hbar}$ plays the role of finite size effects. Finite size scalings are explicit in (44). For $\widehat{\hbar}=1$, we recover the nonsingular model of Section 3.3. For $\hat{\hbar}=0$, we recover the singular radiation model of Section 3.2 (Big Bang). The convergence towards this singular solution as $\widehat{\hbar} \rightarrow 0$ is shown in Figure 8.

3.7. Scalar Field Theory. The phase of inflation in the very early universe is usually described by a scalar field [5]. The ordinary scalar field minimally coupled to gravity evolves according to the equation

$$
\ddot{\phi}+3 H \dot{\phi}+\frac{d V}{d \phi}=0,
$$

where $V(\phi)$ is the potential of the scalar field. The scalar field tends to run down the potential towards lower energies. The density and the pressure of the universe are related to the scalar field by

$$
\rho c^{2}=\frac{1}{2} \dot{\phi}^{2}+V(\phi), \quad p=\frac{1}{2} \dot{\phi}^{2}-V(\phi) .
$$




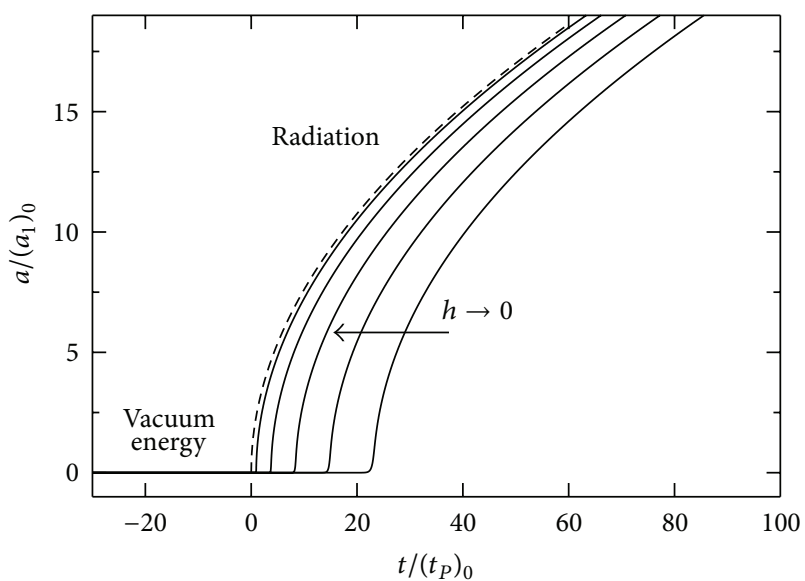

FIgURE 8: Effect of quantum mechanics (finite value of the Planck constant) on the regularization of the singular Big Bang solution ( $\hbar=0$, dashed line) in our simple semi-classical model. The singularity at $t=0$ is replaced by an inflationary expansion from the vacuum energy era to the radiation era. We can draw an analogy with second order phase transitions where the Planck constant plays the role of finite size effects (see the text for details).

Using standard technics [7], we find that the potential corresponding to the equation of state (20) is [34-36]

$$
\begin{gathered}
V(\psi)=\frac{1}{3} \rho_{P} c^{2} \frac{\cosh ^{2} \psi+2}{\cosh ^{4} \psi} \quad(\psi \geq 0), \\
\left(\frac{a}{a_{1}}\right)^{2}=\sinh \psi, \quad \psi=\left(\frac{8 \pi G}{c^{2}}\right)^{1 / 2} \phi .
\end{gathered}
$$

In the vacuum energy era $(t \rightarrow-\infty)$, using (26), we get

$$
\psi \sim\left(\frac{l_{P}}{a_{1}}\right)^{2} e^{\sqrt{32 \pi / 3} t / t_{P}} \longrightarrow 0 .
$$

In the radiation era $(t \rightarrow+\infty)$, using (29), we get

$$
\psi \simeq \ln \frac{t}{t_{P}}+\frac{1}{2} \ln \frac{8 \pi}{3}+2 \ln (2) \longrightarrow+\infty .
$$

More generally, using (31), the evolution of the scalar field $\psi(t)$ in the early universe is given by

$$
\cosh \psi-\ln \left(\frac{1+\cosh \psi}{\sinh \psi}\right)=2\left(\frac{8 \pi}{3}\right)^{1 / 2} \frac{t}{t_{P}}+C .
$$

These results are illustrated in Figures 9 and 10.

\section{The Radiation and the Dark Energy in the Late Universe}

We propose to describe the transition between the radiation era and the dark energy era in the late universe by a single equation of state of the form

$$
p=\frac{1}{3} \rho c^{2}-\frac{4}{3} \rho_{\Lambda} c^{2}
$$

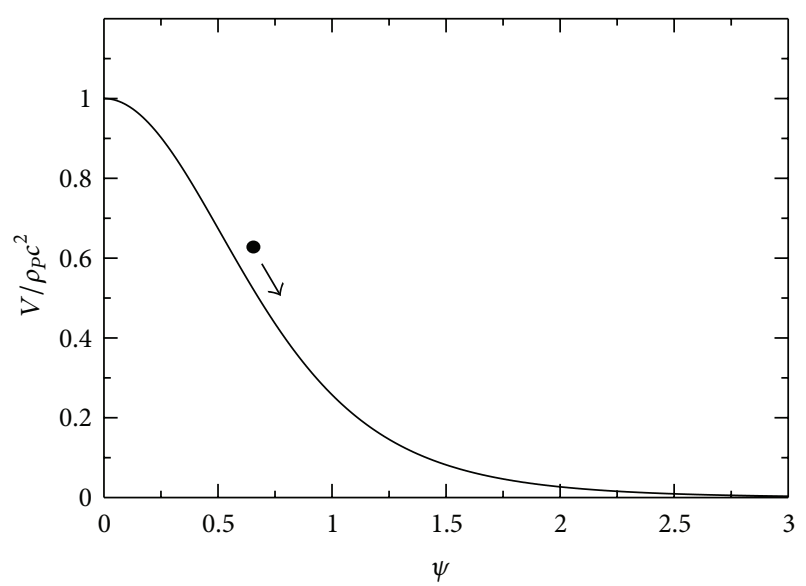

Figure 9: Potential of the scalar field in the early universe. The field tends to run down the potential.

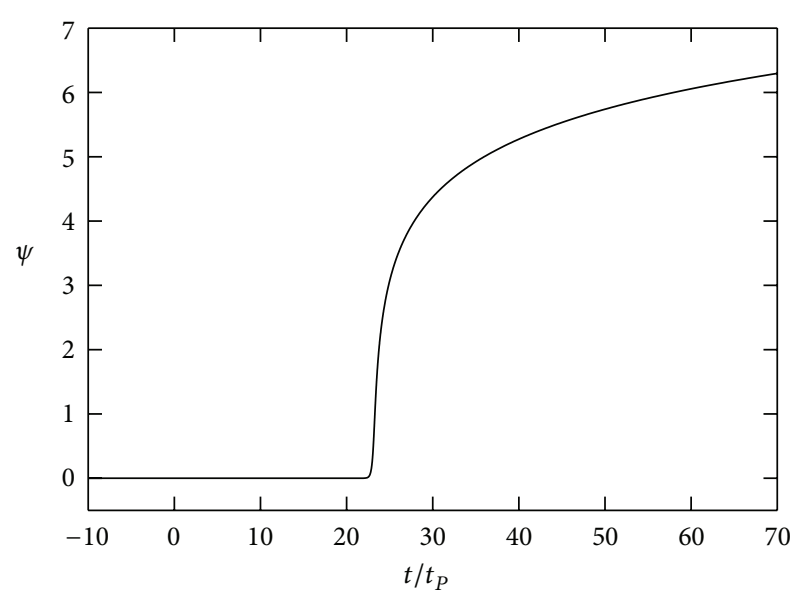

Figure 10: Evolution of the scalar field as a function of time in the early universe. It displays a phase of early inflation.

where $\rho_{\Lambda}=\Lambda / 8 \pi G=7.02 \cdot 10^{-24} \mathrm{~g} / \mathrm{m}^{3}$ is the cosmological density. This equation of state corresponds to a generalized polytropic equation of state (1) with $\alpha=1 / 3, n=-1$, and $k=-(4 / 3) \rho_{\Lambda}$. It may be viewed as the "symmetric" version of the equation of state (20) in the early universe. For $\rho \gg \rho_{\Lambda}$, we recover the equation of state of the pure radiation $p \sim \rho c^{2} / 3$. For $\rho \rightarrow \rho_{\Lambda}$, we get $p \simeq-\rho_{\Lambda} c^{2}$ corresponding to the dark energy.

The continuity equation (15) may be integrated into

$$
\rho=\rho_{\Lambda}\left[\left(\frac{a_{3}}{a}\right)^{4}+1\right]
$$

where $a_{3}$ is a constant of integration. This characteristic scale marks the transition between the radiation era and the dark energy era. The equation of state (51) interpolates smoothly between the radiation era $\left(p=\rho c^{2} / 3\right.$ and $\left.\rho_{\text {rad }} \sim \rho_{\Lambda}\left(a_{3} / a\right)^{4}\right)$ and the dark energy era $\left(p=-\rho c^{2}\right.$ and $\left.\rho=\rho_{\Lambda}\right)$. It provides therefore a "unified" description of radiation and dark energy 
in the late universe. This amounts to summing the density of these two phases. Indeed, (52) may be rewritten as

$$
\rho=\rho_{\text {rad }}+\rho_{\Lambda} \text {. }
$$

At $a=a_{3}$ we have $\rho_{\text {rad }}=\rho_{\Lambda}$ so that $\rho=2 \rho_{\Lambda}$.

4.1. The Dark Energy Era: Late Inflation. When $a \gg a_{3}$, the density tends to a minimum value

$$
\rho=\rho_{\min }=\rho_{\Lambda},
$$

and the pressure tends to $p=-\rho_{\Lambda} c^{2}$. The cosmological density $\rho_{\Lambda}=\Lambda / 8 \pi G=7.02 \cdot 10^{-24} \mathrm{~g} / \mathrm{m}^{3}$ (dark energy) represents a fundamental lower bound for the density. A constant value of the density $\rho \simeq \rho_{\Lambda}$ gives rise to a phase of late inflation. It is convenient to define a cosmological time $t_{\Lambda}=1 /\left(G \rho_{\Lambda}\right)^{1 / 2}=$ $(8 \pi / \Lambda)^{1 / 2}=1.46 \cdot 10^{18} \mathrm{~s}$ and a cosmological length $l_{\Lambda}=c t_{\Lambda}=$ $\left(8 \pi c^{2} / \Lambda\right)^{1 / 2}=4.38 \cdot 10^{26} \mathrm{~m}$. These are the counterparts of the Planck scales for the late universe. From the Friedmann equation (17), we find that the Hubble parameter is constant $H=(8 \pi / 3)^{1 / 2} t_{\Lambda}^{-1}$. Numerically, $H=1.98 \cdot 10^{-18} \mathrm{~s}^{-1}$. Therefore, the scale factor increases exponentially rapidly with time as

$$
a(t) \propto e^{(8 \pi / 3)^{1 / 2} t / t_{\Lambda}} .
$$

This exponential growth corresponds to the de Sitter solution [1]. The timescale of the exponential growth is the cosmological time $t_{\Lambda}=1.46 \cdot 10^{18} \mathrm{~s}$. This solution exists at any time in the future $\left(a \rightarrow+\infty\right.$ and $\rho \rightarrow \rho_{\Lambda}$ for $t \rightarrow+\infty$ ), so there is no future singularity. This is not the case of all cosmological models. In a "phantom universe" [40-57], violating the null dominant energy condition $(w<-1)$, the density increases as the universe expands. This may lead to a future singularity called Big Rip (the density becomes infinite in a finite time). The possibility that we live in a phantom universe is not ruled out by observations.

4.2. The Radiation Era. When $a \ll a_{3}$, we recover the equation

$$
\rho_{\mathrm{rad}} \sim \frac{\rho_{\Lambda} a_{3}^{4}}{a^{4}},
$$

corresponding to the pure radiation described by an equation of state $p=\rho c^{2} / 3$. The conservation of $\rho_{\text {rad }} a^{4}$ implies that $\rho_{\Lambda} a_{3}^{4}=\rho_{\mathrm{rad}, 0} a_{0}^{4}$. Using $\rho_{\Lambda}=\Omega_{\Lambda, 0} \rho_{0}$ and $\rho_{\mathrm{rad}, 0}=\Omega_{\mathrm{rad}, 0} \rho_{0}$ with $\Omega_{\Lambda, 0}=0.763$ (dark energy) and $\Omega_{\mathrm{rad}, 0}=8.48 \cdot 10^{-5}$ (radiation) [1], we obtain $a_{3} / a_{0}=\left(\Omega_{\mathrm{rad}, 0} / \Omega_{\Lambda, 0}\right)^{1 / 4}$ hence

$$
a_{3}=1.36 \cdot 10^{25} \mathrm{~m} \text {. }
$$

This can be rewritten as $a_{3}=0.103 a_{0}=3.09 \cdot 10^{-2} l_{\Lambda}$ where we have used $l_{\Lambda} / a_{0}=H_{0} t_{\Lambda}=3.31$. This scale gives the typical size of the universe at the transition between the radiation era and the dark energy era. When $a \ll a_{3}$, the Friedmann equation (17) yields

$$
\frac{a}{a_{3}} \sim\left(\frac{32 \pi}{3}\right)^{1 / 4}\left(\frac{t}{t_{\Lambda}}\right)^{1 / 2} .
$$

We then have

$$
\frac{\rho}{\rho_{\Lambda}} \sim \frac{3}{32 \pi}\left(\frac{t_{\Lambda}}{t}\right)^{2} .
$$

4.3. The Temperature and the Pressure. The thermodynamical equation (13) with the equation of state (51) may be integrated into

$$
T=T_{P}\left(\frac{15}{\pi^{2}}\right)^{1 / 4}\left(\frac{\rho-\rho_{\Lambda}}{\rho_{P}}\right)^{1 / 4},
$$

where $T_{P}$ is a constant of integration. Using (52), we get

$$
T=T_{P}\left(\frac{15}{\pi^{2}}\right)^{1 / 4}\left(\frac{\rho_{\Lambda}}{\rho_{P}}\right)^{1 / 4} \frac{a_{3}}{a} .
$$

Equation (60) may be seen as a generalized Stefan-Boltzmann law in the late universe. In the radiation era $\rho \gg \rho_{\Lambda}$, it reduces to the ordinary Stefan-Boltzmann law (36). This allows us to identify the constant of integration $T_{P}$ with the Planck temperature $T_{P}=\left(\hbar c^{5} / G k_{B}^{2}\right)^{1 / 2}=1.42 \cdot 10^{32} \mathrm{~K}$. We note that $T \propto a^{-1}$ both in the radiation and dark energy eras. This suggests that radiation and dark energy belong to the same "family". In the radiation era the temperature decreases algebraically as $T \sim T_{P}\left(45 / 32 \pi^{3}\right)^{1 / 4}\left(\rho_{\Lambda} / \rho_{P}\right)^{1 / 4}\left(t_{\Lambda} / t\right)^{1 / 2}$, and in the dark energy era, it decreases exponentially rapidly as $T \propto e^{-(8 \pi / 3)^{1 / 2} t / t_{\Lambda}}$.

The pressure is given by (51). Using (52), we get

$$
p=\left[\frac{1}{3}\left(\frac{a_{3}}{a}\right)^{4}-1\right] \rho_{\Lambda} c^{2} .
$$

It decreases algebraically during the radiation era and tends to a constant negative value $p=-\rho_{\Lambda} c^{2}$ for $t \rightarrow+\infty$. Numerically, $p=-6.31 \cdot 10^{-7} \mathrm{~g} / \mathrm{m} \mathrm{s}^{2}$. The point at which the pressure vanishes is $a_{w}^{\prime}=a_{3} / 3^{1 / 4}, \rho_{w}^{\prime}=4 \rho_{\Lambda}, T_{w}^{\prime}=T_{P}(45 /$ $\left.\pi^{2}\right)^{1 / 4}\left(\rho_{\Lambda} / \rho_{P}\right)^{1 / 4}$. Numerically, $a_{w}^{\prime}=0.760 a_{3}=1.03 \cdot 10^{25} \mathrm{~m}$, $\rho_{w}^{\prime}=4 \rho_{\Lambda}=2.81 \cdot 10^{-23} \mathrm{~g} / \mathrm{m}^{3}, T_{w}^{\prime}=2.81 \cdot 10^{-31} T_{P}=39.9 \mathrm{~K}$.

\section{The General Model}

5.1. The Quadratic Equation of State. We propose to describe the vacuum energy, radiation, and dark energy by a unique equation of state

$$
p_{g}=-\frac{4 \rho_{g}^{2}}{3 \rho_{P}} c^{2}+\frac{1}{3} \rho_{g} c^{2}-\frac{4}{3} \rho_{\Lambda} c^{2} .
$$

For $\rho_{g} \rightarrow \rho_{P}, p_{g} \rightarrow-\rho_{P} c^{2}$ (vacuum energy); for $\rho_{\Lambda} \ll \rho_{g} \ll$ $\rho_{P}, p_{g} \sim \rho_{g} c^{2} / 3$ (radiation); for $\rho_{g} \rightarrow \rho_{\Lambda}, p_{g} \rightarrow-\rho_{\Lambda} c^{2}$ (dark energy). This quadratic equation of state combines the properties of the equation of state (20) valid in the early universe and of the equation of state (51) valid in the late universe. A nice feature of this equation of state is that both the Planck density (vacuum energy) and the cosmological density (dark energy) explicitly appear. Therefore, this equation of state 
reproduces both the early inflation and the late inflation. On the other hand, this description suggests that vacuum energy, radiation, and dark energy may be the manifestation of a unique form of "generalized radiation". This is, however, just a speculation before a justification of this equation of state has been given from first principles. In this paper, we limit ourselves to studying the properties of this equation of state.

Using the equation of continuity (15), we obtain

$$
\rho_{g}=\frac{\rho_{P}}{\left(a / a_{1}\right)^{4}+1}+\rho_{\Lambda}
$$

where $a_{1}=2.61 \cdot 10^{-6} \mathrm{~m}$ (see Section 3). To obtain this expression, we have used the fact that $\rho_{P} \gg \rho_{\Lambda}$ so that $p_{g} / c^{2}+$ $\rho_{g} \simeq\left(4 / 3 \rho_{P}\right)\left(\rho_{g}-\rho_{\Lambda}\right)\left(\rho_{P}-\rho_{g}\right)$. When $a \rightarrow 0, \rho_{g} \rightarrow \rho_{P}$ (vacuum energy); when $\rho_{\Lambda} \ll \rho_{g} \ll \rho_{P}, \rho_{g} \sim \rho_{P}\left(a / a_{1}\right)^{-4}$ (radiation); when $a \rightarrow+\infty, \rho_{g} \rightarrow \rho_{\Lambda}$ (dark energy). In the early universe, the contribution of dark energy is negligible and we recover (21). In the late universe, the contribution of vacuum energy is negligible and we recover (52) with $\rho_{P} a_{1}^{4}=$ $\rho_{\Lambda} a_{3}^{4}$.

We shall view the "generalized radiation", the baryonic matter, and the dark matter as different species. In the general case, these species could interact. However, in this paper, we assume, for simplicity, that they do not. The generalized radiation (vacuum energy + radiation + dark energy) is described by the quadratic equation of state (63). Using $\rho_{P} a_{1}^{4}=\rho_{\text {rad }, 0} a_{0}^{4}$ (see Section 3), we can rewrite (64) in the form

$$
\rho_{g}=\frac{\rho_{\mathrm{rad}, 0}}{\left(a / a_{0}\right)^{4}+\left(a_{1} / a_{0}\right)^{4}}+\rho_{\Lambda}
$$

where $a_{1} / a_{0}=1.97 \cdot 10^{-32}$. The baryonic matter and the dark matter are described as pressureless fluids with $p_{B}=0$ and $p_{\mathrm{DM}}=0$. The equation of continuity (15) for each species leads to the relations

$$
\begin{gathered}
\rho_{B}=\frac{\rho_{B, 0}}{\left(a / a_{0}\right)^{3}}, \\
\rho_{\mathrm{DM}}=\frac{\rho_{\mathrm{DM}, 0}}{\left(a / a_{0}\right)^{3}} .
\end{gathered}
$$

The total density $\rho=\rho_{g}+\rho_{B}+\rho_{\mathrm{DM}}$ of generalized radiation, baryonic matter, and dark energy can be written as

$$
\rho=\frac{\rho_{\mathrm{rad}, 0}}{\left(a / a_{0}\right)^{4}+\left(a_{1} / a_{0}\right)^{4}}+\frac{\rho_{B, 0}}{\left(a / a_{0}\right)^{3}}+\frac{\rho_{\mathrm{DM}, 0}}{\left(a / a_{0}\right)^{3}}+\rho_{\Lambda} .
$$

Substituting this relation in the Friedmann equation (17) and writing $\rho_{\mathrm{rad}, 0}=\Omega_{\mathrm{rad}, 0} \rho_{0}, \rho_{B, 0}=\Omega_{B, 0} \rho_{0}, \rho_{\mathrm{DM}, 0}=\Omega_{\mathrm{DM}, 0} \rho_{0}$, and $\rho_{\Lambda}=\Omega_{\Lambda, 0} \rho_{0}$, we obtain

$$
\frac{H}{H_{0}}=\sqrt{\frac{\Omega_{\mathrm{rad}, 0}}{\left(a / a_{0}\right)^{4}+\left(a_{1} / a_{0}\right)^{4}}+\frac{\Omega_{B, 0}}{\left(a / a_{0}\right)^{3}}+\frac{\Omega_{\mathrm{DM}, 0}}{\left(a / a_{0}\right)^{3}}+\Omega_{\Lambda, 0} .}
$$

From observations, $\Omega_{\mathrm{rad}, 0}=8.48 \cdot 10^{-5}, \Omega_{B, 0}=0.0455$, $\Omega_{\mathrm{DM}, 0}=0.1915, \Omega_{m, 0}=0.237$, and $\Omega_{\Lambda, 0}=0.763$ [1].

The thermodynamic equation (13) with the equation of state (63) may be integrated into

$$
T=T_{P}\left(\frac{15}{\pi^{2}}\right)^{1 / 4}\left(1-\frac{\rho_{g}}{\rho_{P}}\right)^{7 / 4}\left(\frac{\rho_{g}}{\rho_{P}}-\frac{\rho_{\Lambda}}{\rho_{P}}\right)^{1 / 4} .
$$

Using (64), we get

$$
T=T_{P}\left(\frac{15}{\pi^{2}}\right)^{1 / 4} \frac{\left(a / a_{1}\right)^{7}}{\left[\left(a / a_{1}\right)^{4}+1\right]^{2}} .
$$

Equation (70) may also be written as

$$
T=T_{0} \frac{\left(a / a_{0}\right)^{7}}{\left[\left(a / a_{0}\right)^{4}+\left(a_{1} / a_{0}\right)^{4}\right]^{2}},
$$

with $T_{0}=T_{P}\left(15 / \pi^{2}\right)^{1 / 4}\left(a_{1} / a_{0}\right)$. Numerically, $T_{0}=3.11 \mathrm{~K}$. Since $a_{1} \ll a_{0}, T_{0}$ can be identified with the present temperature of radiation. Indeed, after the vacuum energy era, (71) reduces to

$$
T \sim T_{0} \frac{a_{0}}{a} .
$$

Finally, using (63) and (64), the pressure of generalized radiation can be written in very good approximation as

$$
p_{g}=\frac{1}{3} \frac{\left(a / a_{1}\right)^{4}-3}{\left[\left(a / a_{1}\right)^{4}+1\right]^{2}} \rho_{P} c^{2}-\rho_{\Lambda} c^{2}
$$

5.2. The Early Universe. In the early universe, we can neglect the contribution of baryonic matter, dark matter, and dark energy in the density. We only consider the contribution of vacuum energy and radiation. Equation (67) then reduces to

$$
\rho=\frac{\rho_{\text {rad, } 0}}{\left(a / a_{0}\right)^{4}+\left(a_{1} / a_{0}\right)^{4}} .
$$

The Friedmann equation (68) becomes

$$
\frac{H}{H_{0}}=\sqrt{\frac{\Omega_{\mathrm{rad}, 0}}{\left(a / a_{0}\right)^{4}+\left(a_{1} / a_{0}\right)^{4}}} .
$$

It has the analytical solution given by (31). On the other hand, the temperature of the generalized radiation in the early universe is given by (34) and (35). The transition between the vacuum energy era and the radiation era corresponds to $\rho_{\mathrm{rad}}=\rho_{P}$. This yields $a_{1} / a_{0}=\left(\rho_{\mathrm{rad}, 0} / \rho_{P}\right)^{1 / 4}, \rho_{1}=\rho_{P} / 2$, $T_{1}=(1 / 4)\left(15 / \pi^{2}\right)^{1 / 4} T_{P}$. Numerically, $a_{1}=1.97 \cdot 10^{-32} a_{0}=$ $2.61 \cdot 10^{-6} \mathrm{~m}, \rho_{1}=0.5 \rho_{P}=2.58 \cdot 10^{99} \mathrm{~g} / \mathrm{m}^{3}, T_{1}=0.278 T_{P}=$ $3.93 \cdot 10^{31} \mathrm{~K}$. This takes place at a time $t_{1}=23.3 t_{P}=1.25$. $10^{-42} \mathrm{~s}$. This time also corresponds to the inflexion point of the curve $a(t)$. The universe is accelerating for $t<t_{1}$ and decelerating for $t>t_{1}$. 
In the vacuum energy era $\left(a \ll a_{1}\right)$, the density is constant:

$$
\rho \simeq \rho_{P}
$$

Therefore, the Hubble parameter is also a constant $H=$ $(8 \pi / 3)^{1 / 2} t_{P}^{-1}$. In that case, the scale factor and the temperature increase exponentially rapidly according to (26) and (38).

In the radiation era $\left(a \gg a_{1}\right)$, we have

$$
\rho \sim \frac{\rho_{\mathrm{rad}, 0} a_{0}^{4}}{a^{4}} .
$$

The Friedmann equation (75) reduces to

$$
\frac{H}{H_{0}}=\sqrt{\frac{\Omega_{\mathrm{rad}, 0}}{\left(a / a_{0}\right)^{4}}}
$$

and yields

$$
\begin{gathered}
\frac{a}{a_{0}} \sim \Omega_{\mathrm{rad}, 0}^{1 / 4} \sqrt{2 H_{0} t}, \quad \frac{\rho}{\rho_{0}} \sim \frac{1}{\left(2 H_{0} t\right)^{2}}, \\
\frac{T}{T_{0}} \sim \frac{a_{0}}{a} \sim \frac{1}{\Omega_{\mathrm{rad}, 0}^{1 / 4} \sqrt{2 H_{0} t}} .
\end{gathered}
$$

5.3. The Late Universe. In the late universe, we can neglect the contribution of vacuum energy and radiation in the density. We only consider the contribution of baryonic matter, dark matter, and dark energy. Equation (67) then reduces to

$$
\rho=\frac{\rho_{m, 0}}{\left(a / a_{0}\right)^{3}}+\rho_{\Lambda},
$$

where $\rho_{m}=\rho_{B}+\rho_{\mathrm{DM}}$. This coincides with the $\Lambda$ CDM model (see Section 5.5). The Friedmann equation (68) becomes

$$
\frac{H}{H_{0}}=\sqrt{\frac{\Omega_{m, 0}}{\left(a / a_{0}\right)^{3}}+\Omega_{\Lambda, 0}}
$$

with $\Omega_{m, 0}+\Omega_{\Lambda, 0} \simeq 1$. It has the analytical solution

$$
\frac{a}{a_{0}}=\left(\frac{\Omega_{m, 0}}{\Omega_{\Lambda, 0}}\right)^{1 / 3} \sinh ^{2 / 3}\left(\frac{3}{2} \sqrt{\Omega_{\Lambda, 0}} H_{0} t\right) .
$$

The density evolves as

$$
\frac{\rho}{\rho_{0}}=\frac{\Omega_{\Lambda, 0}}{\tanh ^{2}\left((3 / 2) \sqrt{\Omega_{\Lambda, 0}} H_{0} t\right)} .
$$

On the other hand, the temperature of the generalized radiation in the late universe is given by

$$
\begin{gathered}
T=T_{P}\left(\frac{15}{\pi^{2}}\right)^{1 / 4}\left(\frac{\rho_{g}}{\rho_{P}}-\frac{\rho_{\Lambda}}{\rho_{P}}\right)^{1 / 4}, \\
T=T_{0} \frac{a_{0}}{a} .
\end{gathered}
$$

Using the expression (82) of the scale factor, we obtain

$$
T=\frac{T_{0}}{\left(\Omega_{m, 0} / \Omega_{\Lambda, 0}\right)^{1 / 3} \sinh ^{2 / 3}\left((3 / 2) \sqrt{\Omega_{\Lambda, 0}} H_{0} t\right)} .
$$

Finally, the pressure of the generalized radiation in the late universe may be written as

$$
p_{g}=\frac{1}{3} \frac{\rho_{\mathrm{rad}, 0} c^{2}}{\left(a / a_{0}\right)^{4}}-\rho_{\Lambda} c^{2} .
$$

Setting $a=a_{0}$ in (82), we find the age of the universe

$$
t_{0}=\frac{1}{H_{0}} \frac{2}{3} \frac{1}{\sqrt{\Omega_{\Lambda, 0}}} \sinh ^{-1}\left[\left(\frac{\Omega_{\Lambda, 0}}{\Omega_{m, 0}}\right)^{1 / 2}\right] .
$$

Numerically, $t_{0}=1.03 H_{0}^{-1}=0.310 t_{\Lambda}=4.53 \cdot 10^{17} \mathrm{~s} \sim$ 14 Gyrs. It is rather fortunate that the age of the universe almost coincides with the Hubble time $H_{0}^{-1}$.

The universe starts accelerating when $\rho_{m}=2 \rho_{\Lambda}$ [see (16) with $\rho \simeq \rho_{m}+\rho_{\Lambda}$ and $p \simeq-\rho_{\Lambda} c^{2}$. Using $\rho_{m, 0}=\Omega_{m, 0} \rho_{0}$ and $\rho_{\Lambda}=\Omega_{\Lambda, 0} \rho_{0}$ with $\Omega_{m, 0}=0.237$ (matter) and $\Omega_{\Lambda, 0}=0.763$ (dark energy), we obtain $a=a_{c}^{\prime}$ and $t=t_{c}^{\prime}$ with

$$
\frac{a_{c}^{\prime}}{a_{0}}=\left(\frac{\Omega_{m, 0}}{2 \Omega_{\Lambda, 0}}\right)^{1 / 3}, \quad t_{c}^{\prime}=\frac{1}{H_{0}} \frac{2}{3} \frac{1}{\sqrt{\Omega_{\Lambda, 0}}} \sinh ^{-1}\left(\frac{1}{\sqrt{2}}\right) .
$$

At that point $\rho=\rho_{c}^{\prime}=3 \rho_{\Lambda}$ and $T=T_{c}^{\prime}=T_{0} a_{0} / a_{c}^{\prime}$. Numerically, $a_{c}^{\prime}=0.538 a_{0}=0.162 l_{\Lambda}=7.11 \cdot 10^{25} \mathrm{~m}, t_{c}^{\prime}=0.504 H_{0}^{-1}=$ $0.152 t_{\Lambda}=2.22 \cdot 10^{17} \mathrm{~s}, \rho_{c}^{\prime}=3 \rho_{\Lambda}=2.11 \cdot 10^{-23} \mathrm{~g} / \mathrm{m}^{3}$, and $T_{c}^{\prime}=1.86 T_{0}=5.78 \mathrm{~K}$. The universe is decelerating for $t<t_{c}^{\prime}$ and accelerating for $t>t_{c}^{\prime}$.

The transition between the matter era and the dark energy era corresponds to $\rho_{m}=\rho_{\Lambda}$. This leads to $a=a_{2}$ and $t=t_{2}$ with

$$
\frac{a_{2}}{a_{0}}=\left(\frac{\Omega_{m, 0}}{\Omega_{\Lambda, 0}}\right)^{1 / 3}, \quad t_{2}=\frac{1}{H_{0}} \frac{2}{3} \frac{1}{\sqrt{\Omega_{\Lambda, 0}}} \sinh ^{-1}(1) .
$$

At that point $\rho=\rho_{2}=2 \rho_{\Lambda}$ and $T=T_{2}=T_{0} a_{0} / a_{2}$. Numerically, $a_{2}=0.677 a_{0}=0.204 l_{\Lambda}=8.95 \cdot 10^{25} \mathrm{~m}, t_{2}=0.674 H_{0}^{-1}=$ $0.203 t_{\Lambda}=2.97 \cdot 10^{17} \mathrm{~s}, \rho_{2}=2 \rho_{\Lambda}=1.40 \cdot 10^{-23} \mathrm{~g} / \mathrm{m}^{3}$, and $T_{2}=$ $1.48 T_{0}=4.59 \mathrm{~K}$. We also note that $a_{c}^{\prime}=(1 / 2)^{1 / 3} a_{2}=0.794 a_{2}$. Of course, the value

$$
a_{2}=8.95 \cdot 10^{25} \mathrm{~m}
$$

coincides with the one obtained in [34-36] using a different viewpoint.

In the dark energy era $\left(a \gg a_{2}\right)$, the density is constant:

$$
\rho \simeq \rho_{\Lambda} \text {. }
$$

Therefore, the Hubble parameter is also a constant $H=$ $H_{0} \sqrt{\Omega_{\Lambda, 0}}$. Using (82), we find that the scale factor increases exponentially rapidly as

$$
\frac{a}{a_{0}} \sim\left(\frac{\Omega_{m, 0}}{4 \Omega_{\Lambda, 0}}\right)^{1 / 3} e^{\sqrt{\Omega_{\Lambda, 0}} H_{0} t} .
$$


This corresponds to the de Sitter solution. According to (85), the temperature decreases exponentially rapidly as

$$
\frac{T}{T_{0}} \sim\left(\frac{4 \Omega_{\Lambda, 0}}{\Omega_{m, 0}}\right)^{1 / 3} e^{-\sqrt{\Omega_{\Lambda, 0}} H_{0} t} .
$$

In the matter era $\left(a \ll a_{2}\right)$, we have

$$
\rho \sim \frac{\rho_{m, 0} a_{0}^{3}}{a^{3}} .
$$

The Friedmann equation (81) reduces to

$$
\frac{H}{H_{0}}=\sqrt{\frac{\Omega_{m, 0}}{\left(a / a_{0}\right)^{3}}},
$$

and we have

$$
\begin{gathered}
\frac{a}{a_{0}}=\Omega_{m, 0}^{1 / 3}\left(\frac{3}{2} H_{0} t\right)^{2 / 3}, \quad \frac{\rho}{\rho_{0}}=\frac{1}{\left((3 / 2) H_{0} t\right)^{2}}, \\
\frac{T}{T_{0}} \sim \frac{1}{\Omega_{m, 0}^{1 / 3}\left((3 / 2) H_{0} t\right)^{2 / 3}} .
\end{gathered}
$$

This corresponds to the Einstein-de Sitter (EdS) universe.

5.4. The Evolution of the Late Universe. In the matter era $(a \ll$ $a_{2}$ ), the radius increases algebraically as $a \propto t^{2 / 3}$, while the density decreases algebraically as $\rho \propto t^{-2}$ (EdS). When $a \gg$ $a_{2}$, the universe enters in the dark energy era. It undergoes a late inflation (de Sitter) during which its radius increases exponentially rapidly, while its density remains constant and equal to the cosmological density $\rho_{\Lambda}$. The transition takes place at $a_{2}=0.677 a_{0}=0.204 l_{\Lambda}=8.95 \cdot 10^{25} \mathrm{~m}, \rho_{2}=2 \rho_{\Lambda}=$ $1.40 \cdot 10^{-23} \mathrm{~g} / \mathrm{m}^{3}, T_{2}=1.48 T_{0}=4.59 \mathrm{~K}$, and $t_{2}=0.674 H_{0}^{-1}=$ $0.203 t_{\Lambda}=2.97 \cdot 10^{17} \mathrm{~s}$. In the matter era, the universe is decelerating, and in the dark energy era, it is accelerating. The time at which the universe starts accelerating is $t_{c}^{\prime}=$ $0.504 H_{0}^{-1}=0.152 t_{\Lambda}=2.22 \cdot 10^{17} \mathrm{~s}$, corresponding to a radius $a_{c}^{\prime}=0.538 a_{0}=0.162 l_{\Lambda}=0.794 a_{2}=7.11 \cdot 10^{25} \mathrm{~m}$, a density $\rho_{c}^{\prime}=3 \rho_{\Lambda}=2.11 \cdot 10^{-23} \mathrm{~g} / \mathrm{m}^{3}$ and a temperature $T_{c}^{\prime}=1.86 T_{0}=$ $5.78 \mathrm{~K}$. In the late universe, the temperature of generalized radiation always scales as $T \propto a^{-1}$. It decreases algebraically rapidly $\left(T \propto t^{-2 / 3}\right)$ in the matter era and exponentially rapidly in the dark energy era. The evolution of the scale factor, density, temperature, and pressure of radiation as a function of time is represented in Figures 11, 12, 13, 14, 15, and 16 in logarithmic and linear scales.

The present size of the universe $a_{0}=0.302 l_{\Lambda}=1.32$. $10^{26} \mathrm{~m}$ is precisely of the order of the scale $a_{2}=0.204 l_{\Lambda}=$ $8.95 \cdot 10^{25} \mathrm{~m}\left(a_{0}=1.48 a_{2}\right)$. We have $a_{0} \sim a_{2} \sim l_{\Lambda}$ and $t_{0} \sim t_{2} \sim t_{\Lambda}$. Therefore, we live just at the transition between the matter era and the dark energy era (see bullets in Figures $11,12,13,14,15$, and 16). (In the context of the standard $\Lambda \mathrm{CDM}$ model, the way to state this observation is to say that the present ratio $\Omega_{\Lambda, 0} / \Omega_{m, 0}=3.22$ between dark energy and matter is of order unity. Since the matter density changes

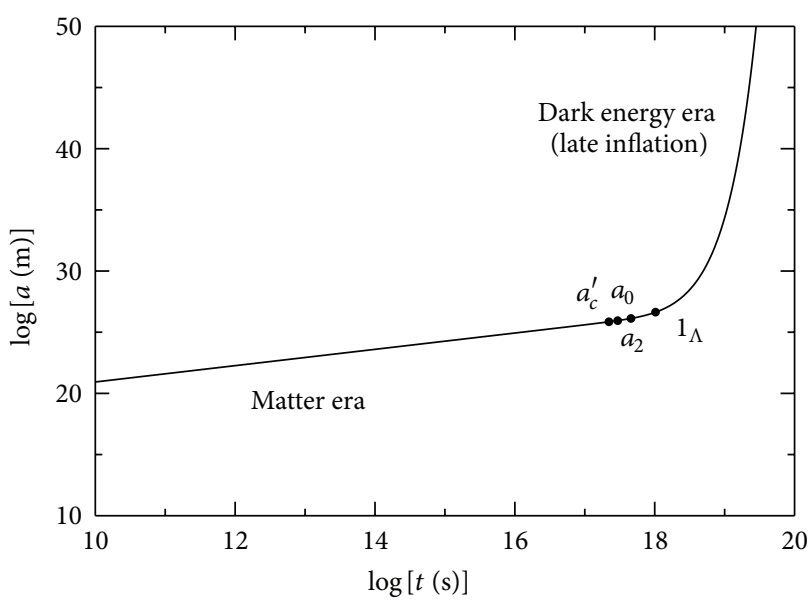

Figure 11: Evolution of the scale factor $a$ with the time $t$ in logarithmic scales. This figure clearly shows the transition between the matter era (EdS) and the dark energy era (de Sitter). In the dark energy era, the radius increases exponentially rapidly on a timescale of the order of the cosmological time $t_{\Lambda}=1.46 \cdot 10^{18} \mathrm{~s}$. This corresponds to a phase of late inflation. The universe is decelerating for $a<a_{c}^{\prime}$ and accelerating for $a>a_{c}^{\prime}$ with $a_{c}^{\prime}=7.11 \cdot 10^{25} \mathrm{~m}$. The transition between the matter era and the dark energy era takes place at $a_{2}=8.95 \cdot 10^{25} \mathrm{~m}$. Coincidentally, the present universe turns out to be close to the transition point $\left(a_{0} \sim a_{2}\right)$.

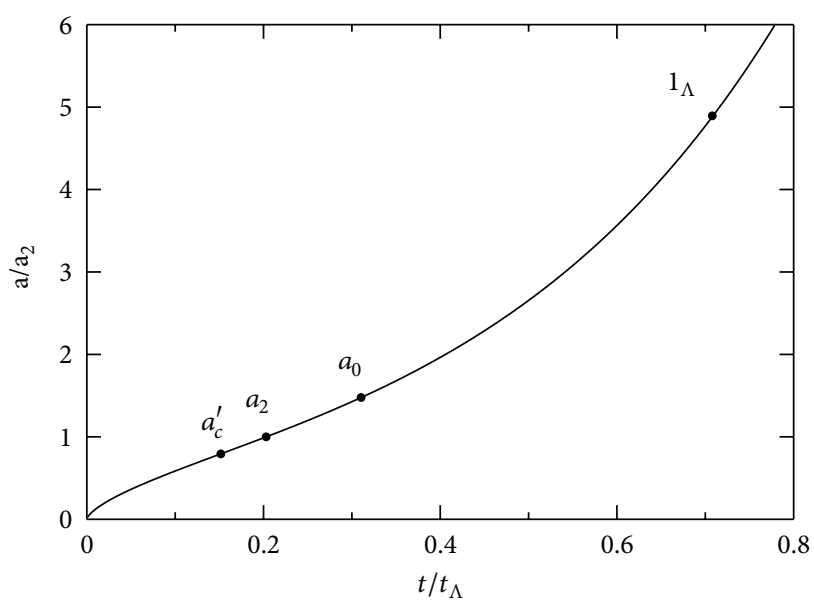

FIGURE 12: Evolution of the scale factor $a$ with the time $t$ in linear scales.

as $a^{-3}$ the ratio between matter and dark energy is of order unity only during a brief period of time in the evolution of the universe. It turns out that we live precisely in this period. This coincidence is intriguing and often referred to as the "cosmic coincidence problem" [58]. Several theories have been proposed to explain why $\Omega_{\Lambda, 0} / \Omega_{m, 0} \sim 1$ [59-61]. However, this may be just a pure coincidence without deeper reason. Life (and researchers inquiring about cosmology) may have emerged at this special moment of the history of the universe, 14 Gyrs after the Big Bang, precisely at the epoch where $\Omega_{\Lambda} / \Omega_{m} \sim 1$. Life cannot have emerged much earlier because galaxies, stars, and planets are not created, and it 


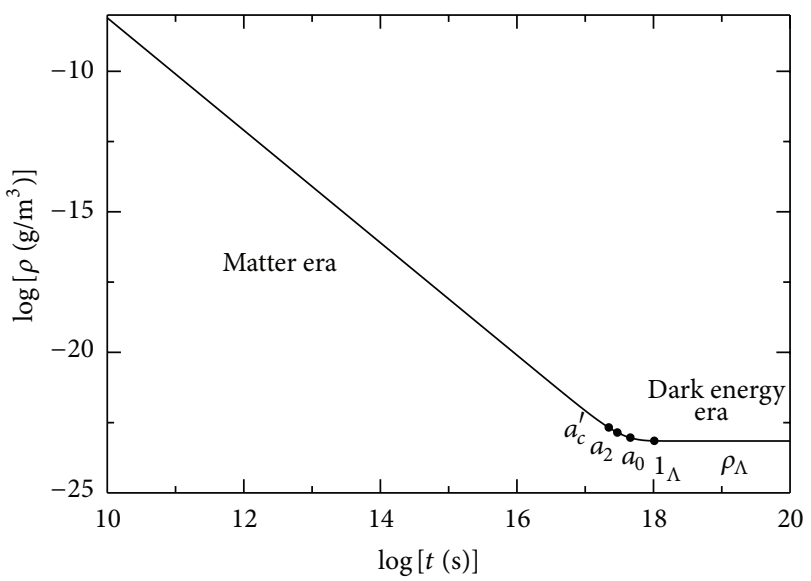

FIGURE 13: Evolution of the density $\rho$ with the time $t$ in logarithmic scales. During the late inflation, the density remains approximately constant with the cosmological value $\rho_{\min }=\rho_{\Lambda}$ representing a lower bound.

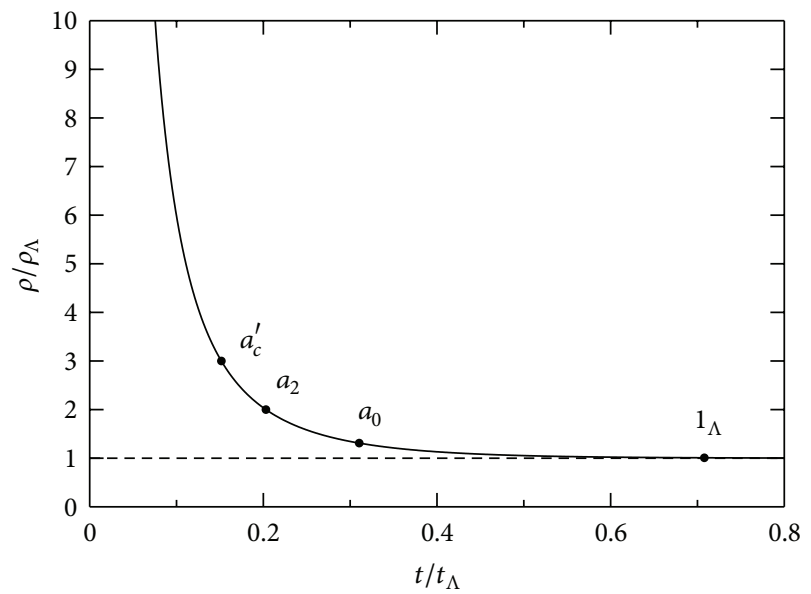

FIGURE 14: Evolution of the density $\rho$ with the time $t$ in linear scales. General relativity (cosmological constant) limits the decay of the density to the cosmological value $\rho_{\Lambda}=7.02 \cdot 10^{-24} \mathrm{~g} / \mathrm{m}^{3}$.

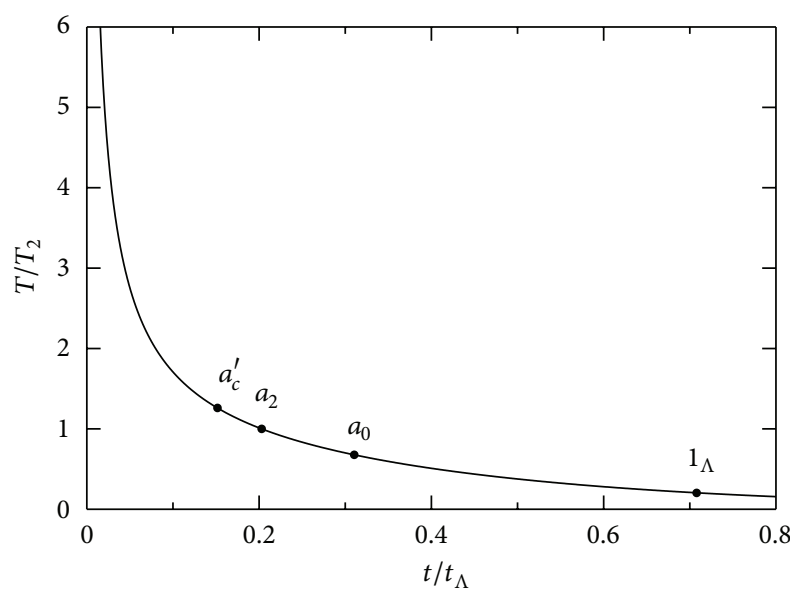

FIgURE 15: Evolution of the temperature of radiation $T$ with the time $t$ in linear scales.

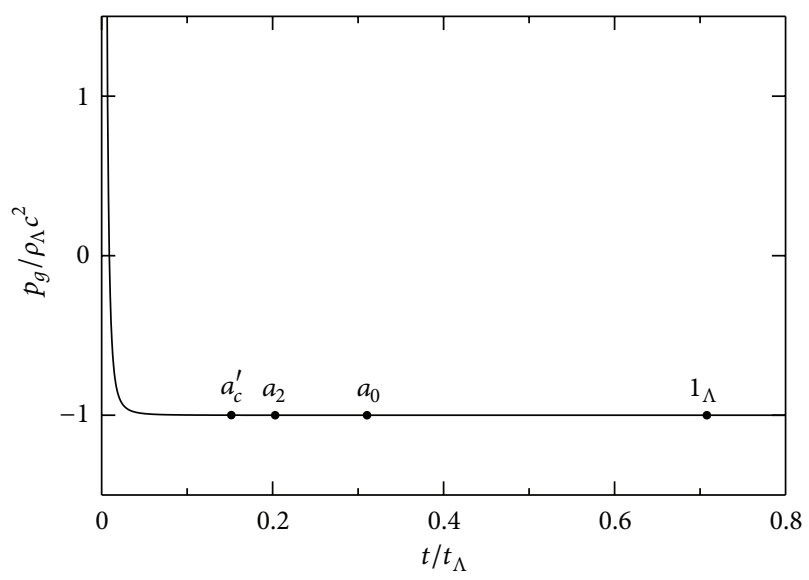

FIGURE 16: Evolution of the pressure of radiation $p_{g}$ with the time $t$ in linear scales.

may not persist much later because planets, stars, and galaxies will die. Therefore, this coincidence is not really a "problem.") The present density of the universe is $\rho_{0}=1.31 \rho_{\Lambda}=9.20$. $10^{-24} \mathrm{~g} / \mathrm{m}^{3}$ and the age of the universe is $t_{0}=1.03 H_{0}^{-1}=$ $0.310 t_{\Lambda}=4.54 \cdot 10^{17} \mathrm{~s} \sim 14$ Gyrs. The present values of the equation of state parameter and deceleration parameter are $w_{0}=-\Omega_{\Lambda, 0}$ and $q_{0}=\left(1-3 \Omega_{\Lambda, 0}\right) / 2\left[\right.$ since $p \simeq-\rho_{\Lambda} c^{2}$ ]. Numerically, $w_{0}=-0.763$ and $q_{0}=-0.645$.

5.5. The Whole Evolution of the Universe. In the standard $\Lambda \mathrm{CDM}$ model, the radiation, baryonic matter, dark matter, and dark energy are treated as four independent species characterized by different equations of state. The equation of state is $p=\rho c^{2} / 3$ for the radiation, $p=0$ for the baryonic matter and dark matter, and $p=-\rho c^{2}$ for the dark energy. One then solves the equation of continuity (15) for these four species in order to obtain the relation between their density and the scale factor, that is, $\rho_{\mathrm{rad}}=\rho_{\mathrm{rad}, 0} /\left(a / a_{0}\right)^{4}, \rho_{B}=$ $\rho_{B, 0} /\left(a / a_{0}\right)^{3}, \rho_{\mathrm{DM}}=\rho_{\mathrm{DM}, 0} /\left(a / a_{0}\right)^{3}$, and $\rho_{\Lambda}=\rho_{\Lambda, 0}$. Finally, we add their individual densities $\rho=\rho_{\mathrm{rad}}+\rho_{B}+\rho_{\mathrm{DM}}+\rho_{\Lambda}$, that is,

$$
\rho=\frac{\rho_{\mathrm{rad}, 0}}{\left(a / a_{0}\right)^{4}}+\frac{\rho_{B, 0}}{\left(a / a_{0}\right)^{3}}+\frac{\rho_{\mathrm{DM}, 0}}{\left(a / a_{0}\right)^{3}}+\rho_{\Lambda, 0}
$$

and solve the Friedmann equation (17). This leads to the equation

$$
\frac{H}{H_{0}}=\sqrt{\frac{\Omega_{\mathrm{rad}, 0}}{\left(a / a_{0}\right)^{4}}+\frac{\Omega_{B, 0}}{\left(a / a_{0}\right)^{3}}+\frac{\Omega_{\mathrm{DM}, 0}}{\left(a / a_{0}\right)^{3}}+\Omega_{\Lambda, 0},}
$$

with $\Omega_{\mathrm{rad}}+\Omega_{B}+\Omega_{\mathrm{DM}}+\Omega_{\Lambda}=1$. This standard model provides a good description of the universe after the Planck era. However, it exhibits a primordial singularity at $t=0$ (Big Bang). Furthermore, it does not describe the phase of early inflation. This phase is usually described by another theory, for example, a scalar field theory, and it is then connected to the standard model.

In the present model, only three species coexist: baryonic matter (see (66a)), dark matter (see (66b)), and a "generalized 
radiation" unifying vacuum energy, radiation, and dark energy (see (65)). As a result, the equation describing the whole evolution of the universe is (In the standard model, the radiation density diverges as $a^{-4}$ when $a \rightarrow 0$ while the matter density diverges as $a^{-3}$ which is subdominant. Therefore, we can safely extrapolate the matter density to $a=0$ (its contribution is negligible anyway). In the present model, the generalized radiation density (65) does not diverge anymore when $a \rightarrow 0$ since $a_{1} \neq 0$. Therefore, it becomes necessary to state explicitly that matter appears at sufficiently late time in order to avoid a spurious divergence of the matter density when $a \rightarrow 0$. To that purpose, it is sufficient to add a Heaviside function $H\left(a-a_{1}\right)$ in the matter density and write $\left.\Omega_{m, 0}^{*}=\Omega_{m, 0} H\left(a-a_{1}\right).\right)$

$$
\frac{H}{H_{0}}=\sqrt{\frac{\Omega_{\mathrm{rad}, 0}}{\left(a / a_{0}\right)^{4}+\left(a_{1} / a_{0}\right)^{4}}+\frac{\Omega_{B, 0}^{*}}{\left(a / a_{0}\right)^{3}}+\frac{\Omega_{\mathrm{DM}, 0}^{*}}{\left(a / a_{0}\right)^{3}}+\Omega_{\Lambda, 0}},
$$

with $\Omega_{B}+\Omega_{\mathrm{rad}}+\Omega_{\mathrm{DM}}+\Omega_{\Lambda}=1$. For $a_{1}=0$, we obtain the same equation as in the standard $\Lambda$ CDM model where the contributions of radiation, baryonic matter, dark matter, and dark energy are added individually [1]. However, as we have previously mentioned, the $\Lambda \mathrm{CDM}$ model does not describe the phase of early inflation and it presents a singularity at $t=0$ (Big Bang). For $a_{1} / a_{0}=1.97 \cdot 10^{-32}$, we obtain a generalized model which does not present a primordial singularity and which displays a phase of early inflation. In this model, the universe always exists in the past but, for $t<0$, it has a very small radius, smaller than the Planck length. (As already stated in Section 3, our model certainly breaks down in this period since it does not take quantum fluctuations into account. The Planck era may not be described in terms of an equation of state $p(\rho)$ or even in terms of the Einstein equations, as we have assumed. It probably requires the development of a theory of quantum gravity that does not exist for the moment. An interesting description of the early inflation has been given by Monerat et al. [62] in terms of a quantized model based on a simplified Wheeler-DeWitt equation. In that model, a quantum tunneling process explains the birth of the universe with a well-defined size after tunneling. Therefore, other inflationary scenarios are possible in addition to the one based on the generalized equation of state (63).) At $t=0$, it undergoes an inflationary expansion in a very short lapse of time of the order of 23.3 Planck times. For $t>23.3 t_{P}$, this model gives the same results as the standard $\Lambda \mathrm{CDM}$ model: the universe first undergoes an algebraic expansion in the radiation and matter eras, then an exponential expansion (second inflation) in the dark energy era. A nice feature of this model is its simplicity since it incorporates a phase of early inflation in a very simple manner. We just have to add a term $+\left(a_{1} / a_{0}\right)^{4}$ in the standard equation (99). Therefore, the modification implied by (65) to describe the early inflation is very natural. On the other hand, this model gives the same results in the late universe as the standard model, so this does not bring any modification to the usual equation (99). Therefore, our simple model completes the standard
$\Lambda \mathrm{CDM}$ model by incorporating the phase of early inflation in a natural manner. This is an interest of this description since the standard model gives results that agree with observations at late times.

The evolution of the scale factor with time is obtained by solving the first-order differential equation (100). This yields

$$
\begin{aligned}
& \int_{a_{i} / a_{0}}^{a / a_{0}} d x\left(x \sqrt{\frac{\Omega_{\mathrm{rad}, 0}}{x^{4}+\left(a_{1} / a_{0}\right)^{4}}+\frac{\Omega_{B, 0}^{*}}{x^{3}}+\frac{\Omega_{\mathrm{DM}, 0}^{*}}{x^{3}}+\Omega_{\Lambda, 0}}\right)^{-1} \\
& =H_{0} t
\end{aligned}
$$

where $a_{i}=a_{1}=0$ in the standard model and $a_{i}=l_{P}$ in the generalized model. The age of the universe is

$$
\begin{aligned}
t_{0}= & \frac{1}{H_{0}} \\
& \times \int_{0}^{1} \frac{d x}{x \sqrt{\left(\Omega_{\mathrm{rad}, 0} / x^{4}\right)+\left(\Omega_{B, 0} / x^{3}\right)+\left(\Omega_{\mathrm{DM}, 0} / x^{3}\right)+\Omega_{\Lambda, 0}}} .
\end{aligned}
$$

Of course, for the determination of the age of the universe, we can neglect the contribution of vacuum energy in the early universe and take $a_{1}=0$ (strictly speaking, the age of the universe is infinite since it has no origin; however, we define the age of the universe from the time $t=0$ at which $a=l_{P}$ ). We obtain the standard result $t_{0}=1.03 \mathrm{H}_{0}^{-1}=$ $4.53 \cdot 10^{17} \mathrm{~s}=14.4 \mathrm{Gyr}$. (The Hubble constant is usually written as $H_{0}=2.268 h_{7} 10^{-18} \mathrm{~s}^{-1}$ where the dimensionless parameter $h_{7}$ is about $10 \%$ of unity [1]. For simplicity, we have taken $h_{7}=1$ in the numerical applications. The current value is $h_{7}=1.05 \pm 0.05$. If we take $h_{7}=1.05$, the age of the universe is $t_{0}=13.7 \mathrm{Gyr}$.) Actually, we find the same result if we neglect radiation and use the analytical expression (87) instead of (102).

In Figures 17-19, we have represented the evolution of the radius, density, and temperature of the universe as a function of time. The universe exhibits two types of inflations: an early inflation due to the Planck density $\rho_{P}=5.16 \cdot 10^{99} \mathrm{~g} / \mathrm{m}^{3}$ (vacuum energy) and a late inflation due to the cosmological density $\rho_{\Lambda}=7.02 \cdot 10^{-24} \mathrm{~g} / \mathrm{m}^{3}$ (dark energy). There exists a striking "symmetry" between the early and the late evolution of the universe, and the cosmological constant in the late universe plays the same role as the Planck constant in the early universe. In particular, Figure 18 shows that the density varies between two bounds $\rho_{\max }=\rho_{P}$ and $\rho_{\min }=\rho_{\Lambda}$ that are fixed by fundamental constants. These values differ by a factor of the order $10^{122}$. The early universe is governed by quantum mechanics $(\hbar)$ and the late universe by general relativity $(\Lambda)$.

Remark 1. We have described dark matter as a presureless fluid with $p=0$. There are some indications that dark matter may be described by an isothermal equation of state $p=\alpha \rho c^{2}$ [63]. In that case, the term $\Omega_{\mathrm{DM}, 0}^{*} /\left(a / a_{0}\right)^{3}$ in (100) should be replaced by $\Omega_{\mathrm{DM}, 0}^{*} /\left(a / a_{0}\right)^{3(1+\alpha)}$. Another generalization 


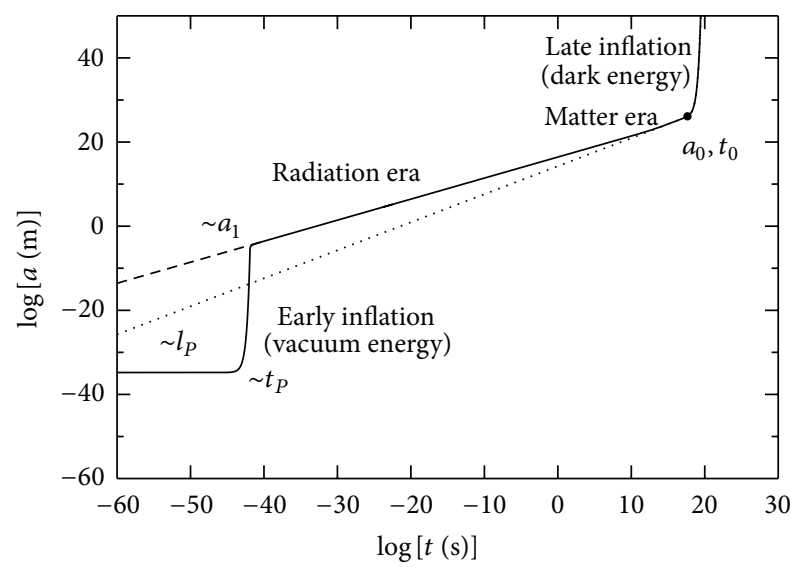

FIGURE 17: Evolution of the scale factor $a$ as a function of time in logarithmic scales. The early universe undergoes a phase of inflation due to the vacuum energy before entering into the radiation era. During the early inflation, the scale factor increases by 29 orders of magnitude in less than $10^{-42} \mathrm{~s}$. This is followed by the matter era and the dark energy era responsible for the accelerated expansion of the universe. The universe exhibits two types of inflation: an early inflation corresponding to the Planck density $\rho_{P}$ (vacuum energy) due to quantum mechanics (Planck constant) and a late inflation corresponding to the cosmological density $\rho_{\Lambda}$ (dark energy) due to general relativity (cosmological constant). The evolution of the early and late universe is remarkably symmetric. We have represented in dashed line the standard model leading to a primordial singularity (Big Bang). The dotted line corresponds to the model of Section 5.3 where the radiation is neglected. This analytical model provides a good description of the late universe. We have also represented the location of the present universe (bullet). It happens to be just at the transition between the matter era and the dark energy era.

may be introduced if dark matter is made of Bose-Einstein condensates (BECs) instead of being pressureless. In that case, the term $\Omega_{\mathrm{DM}, 0}^{*} /\left(a / a_{0}\right)^{3}$ in (100) should be replaced by $\Omega_{\mathrm{DM}, 0}^{*} /\left[\left(a / a_{0}\right)^{3} \mp\left(a_{\mathrm{BEC}} / a_{0}\right)^{3}\right]$ as proposed in $[64,65]$. It is shown in these papers that the presence of BECs can substantially accelerate the formation of the large-scale structures of the universe.

\section{Conclusion}

There are different manners to describe the evolution of the universe.

In the standard $\Lambda \mathrm{CDM}$ model [1], the radiation, baryonic matter, dark matter, and dark energy are treated as four independent species characterized by different equations of state. The equation of state is $p=\rho c^{2} / 3$ for the radiation, $p=0$ for the baryonic matter and dark matter, and $p=$ $-\rho c^{2}$ for the dark energy. One then solves the equation of continuity (15) for each species in order to obtain the relation between their density and the scale factor. Finally, we add their densities $\rho=\rho_{\mathrm{rad}}+\rho_{B}+\rho_{\mathrm{DM}}+\rho_{\Lambda}$ and solve the Friedmann equation (17). This leads to (99). This standard model provides a good description of the universe after the Planck era. However, it exhibits a primordial singularity (Big Bang) and does not display a phase of early inflation.

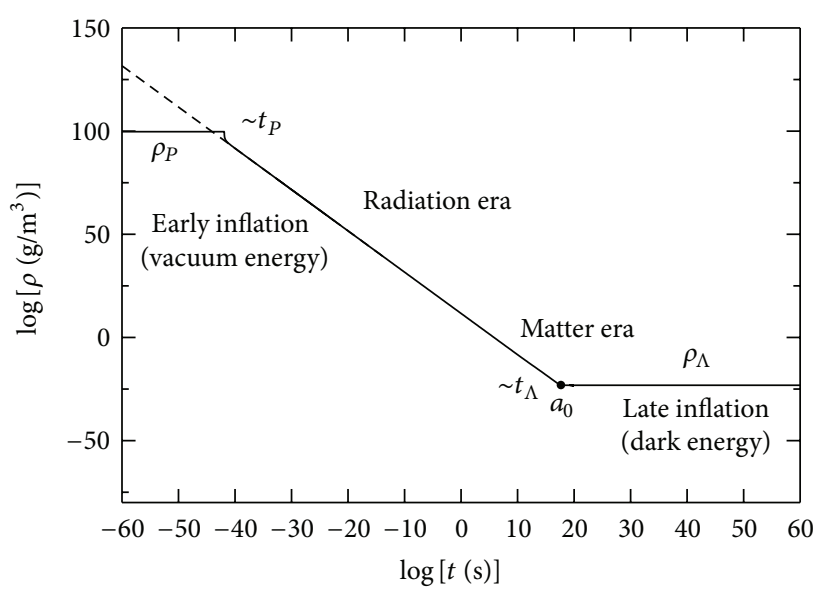

FIGURE 18: Evolution of the density $\rho$ as a function of time in logarithmic scales. The density goes from a maximum value $\rho_{\max }=$ $\rho_{P}$ determined by the Planck constant (quantum mechanics) to a minimum value $\rho_{\min }=\rho_{\Lambda}$ determined by the cosmological constant (general relativity). These two bounds, which are fixed by fundamental constants of physics, are responsible for the early and late inflation of the universe. In between, the density decreases as $t^{-2}$.

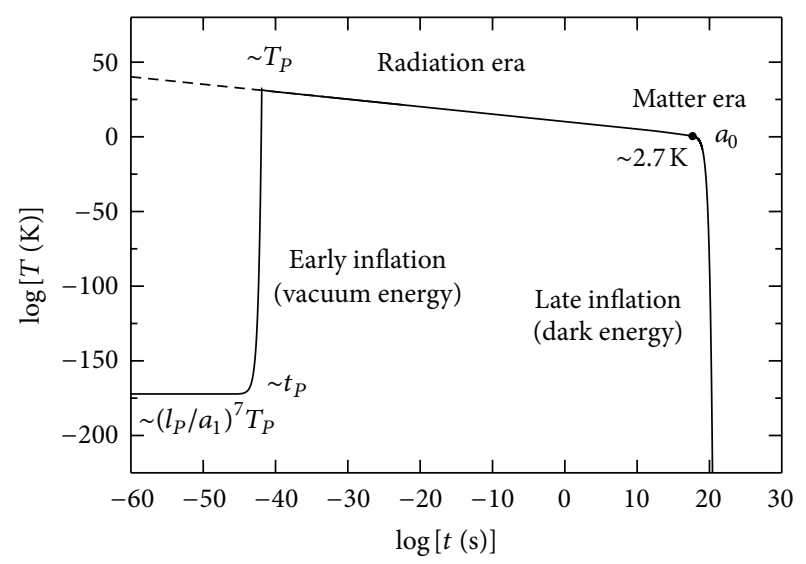

FIGURE 19: Evolution of the temperature $T$ of the "generalized" radiation as a function of time in logarithmic scales. Before the early inflation, the universe is extremely cold $\left(T<10^{-173} \mathrm{~K}\right)$. During the early inflation, the temperature increases by 204 orders of magnitude in less than $10^{-42} \mathrm{~s}$. During the radiation era, the temperature decreases algebraically. The present value of the temperature is $T \simeq$ $2.7 \mathrm{~K}$ (bullet). During the late inflation, the temperature decreases exponentially rapidly.

In recent papers [34-36], we have proposed a different description. We have described the early universe (vacuum energy + radiation) and the late universe (matter + dark energy) by two symmetric equations of state. The transition between the vacuum energy era and the radiation era in the early universe has been described by a single equation of state of the form $p=-(4 / 3)\left(\rho^{2} / \rho_{P}\right) c^{2}+\rho c^{2} / 3$. Similarly, the transition between the matter era and the dark energy era in the late universe has been described by a single equation of state $p=-\rho_{\Lambda} c^{2}$. These equations of state may be viewed 
as two polytropic equations of state with index $n=1$ and $n=-1$, respectively. This description leads to very symmetric mathematical formulae in the early and late universe; the cosmological density in the late universe plays a role similar to that of the Planck density in the early universe. In order to obtain a single equation describing the whole evolution of the universe, we have just added the density of these two phases $\rho=\rho_{\text {early }}+\rho_{\text {late }}$ in the Friedmann equation (17). This leads to $(100)$ with $a_{1} / a_{0}=1.97 \cdot 10^{-32}$. For $t>23.3 t_{P}$, the evolution is the same as in the $\Lambda$ CDM model. However, this model incorporates a phase of early inflation in a natural manner and yields a model without primordial singularity (although we should not extrapolate it in the infinite past due to our neglect of quantum fluctuations). This may be called "aioniotic universe" since it has no origin nor end. As mentioned in the Introduction, this model may be viewed as a generalization of the Chaplygin gas model.

Finally, in this paper, we have adopted a different point of view. We have described the vacuum energy + radiation + dark energy as a single species called "generalized radiation" characterized by a quadratic equation of state $p_{g}=$ $-(4 / 3)\left(\rho_{g}^{2} / \rho_{P}\right) c^{2}+\rho_{g} c^{2} / 3-\rho_{\Lambda} c^{2}$. From this equation of state, we have obtained a generalization of the Stefan-Boltzmann law (see (69)). The baryonic matter and dark matter are treated as different species with a pressureless equation of state $p_{B}=p_{\mathrm{DM}}=0$. We have solved the equation of continuity (15) to obtain the relation between the density and the scale factor for these three species. In order to obtain a single equation describing the whole evolution of the universe, we have just added their densities $\rho=\rho_{g}+\rho_{B}+\rho_{\mathrm{DM}}$ in the Friedmann equation (17). This again leads to (100), as in our previous approach. However, the point of view is different. The present approach suggests that vacuum energy and dark energy may correspond to a form of "generalized radiation." This may open new perspectives in cosmology. Of course, more work is needed to justify this quadratic equation of state. However, the present paper shows that this equation of state is able to reproduce the main properties of our universe.

\section{Appendices}

\section{A. The Intermediate Universe: Transition between the Radiation Era and the Matter Era}

In this Appendix, we consider the epoch where the evolution of the universe is dominated by radiation and matter. The total density can be written as

$$
\rho=\frac{\rho_{\mathrm{rad}, 0}}{\left(a / a_{0}\right)^{4}}+\frac{\rho_{m, 0}}{\left(a / a_{0}\right)^{3}} .
$$

The transition between the radiation era and the matter era takes place when $\rho_{\text {rad }}=\rho_{m}$ leading to $a=a_{4}=$ $\left(\Omega_{\mathrm{rad}, 0} / \Omega_{m, 0}\right) a_{0}$. Numerically, $a_{4}=3.58 \cdot 10^{-4} a_{0}=1.08$. $10^{-4} l_{\Lambda}$, hence

$$
a_{4}=4.73 \cdot 10^{22} \mathrm{~m}
$$

This corresponds to a density $\rho_{4}=2\left(\Omega_{m, 0}^{4} / \Omega_{\text {rad, } 0}^{3}\right) \rho_{0}$ and a temperature $T_{4}=T_{0} a_{0} / a_{4}$. Numerically, $\rho_{4}=1.03 \cdot 10^{10} \rho_{0}=$ $1.36 \cdot 10^{10} \rho_{\Lambda}=9.52 \cdot 10^{-14} \mathrm{~g} / \mathrm{m}^{3}$ and $T_{4}=2.79 \cdot 10^{3} T_{0}=$ $8.69 \cdot 10^{3} \mathrm{~K}$.

The Friedmann equation (100) simplifies into

$$
\frac{H}{H_{0}}=\sqrt{\frac{\Omega_{\mathrm{rad}, 0}}{\left(a / a_{0}\right)^{4}}+\frac{\Omega_{m, 0}}{\left(a / a_{0}\right)^{3}}} .
$$

The evolution of the scale factor with time is obtained by solving the first-order differential equation (A.3). This yields

$$
\int_{0}^{a / a_{0}} \frac{d x}{x \sqrt{\left(\Omega_{\mathrm{rad}, 0} / x^{4}\right)+\left(\Omega_{m, 0} / x^{3}\right)}}=H_{0} t .
$$

The integral can be performed analytically leading to

$$
\begin{aligned}
H_{0} t= & \frac{4}{3} \frac{\left(\Omega_{\mathrm{rad}, 0}\right)^{3 / 2}}{\left(\Omega_{m, 0}\right)^{2}} \\
& -\frac{2}{3} \frac{1}{\left(\Omega_{m, 0}\right)^{1 / 2}}\left(\frac{2 \Omega_{\mathrm{rad}, 0}}{\Omega_{m, 0}}-\frac{a}{a_{0}}\right) \sqrt{\frac{\Omega_{\mathrm{rad}, 0}}{\Omega_{m, 0}}+\frac{a}{a_{0}} .}
\end{aligned}
$$

In the radiation era $\left(a \ll a_{4}\right)$, we recover (79), and in the matter era $\left(a \gg a_{4}\right)$, we recover (96). The transition between the radiation era and the matter era takes place at a time $H_{0} t_{4}=(1 / 3)(4-2 \sqrt{2})\left(\Omega_{\mathrm{rad}, 0}\right)^{3 / 2} /\left(\Omega_{m, 0}\right)^{2}$. Numerically, $t_{4}=$ $5.43 \cdot 10^{-6} H_{0}^{-1}=1.64 \cdot 10^{-6} t_{\Lambda}=2.39 \cdot 10^{12} \mathrm{~s}$.

\section{B. Analytical Solution of the Friedmann Equations for a Toy Universe without Matter}

For the sake of completeness, we give the solution of the Friedmann equations for a universe described by the pure equation of state (63). This solution is interesting mathematically because it is analytical, and it exhibits two phases of inflation separated by a radiation era. However, it is unphysical in the intermediate phase because it ignores the contribution of baryonic and dark matter. The numerical solution of (100) studied in the core of the paper is more physical.

In the early universe, the equation of state (63) reduces to $(20)$. The density is given as a function of the scale factor by (21). The evolution of the scale factor is given by (31). This solution is satisfactory physically since matter can be neglected in the early universe.

In the late universe, the equation of state (63) reduces to (51). The density is given as a function of the scale factor by (52). If we neglect the contribution of matter, the solution of the Friedmann equation (17) with the density (52) is

$$
\frac{a}{a_{3}}=\sinh ^{1 / 2}\left[\left(\frac{32 \pi}{3}\right)^{1 / 2} \frac{t}{t_{\Lambda}}\right] .
$$

For $t \rightarrow 0$, it reduces to (58), and for $t \rightarrow+\infty$, we obtain (55) with a prefactor $1 / \sqrt{2}$. Furthermore, we can associate to 
the equation of state (51) a scalar field as in Section 3.7. The potential of this scalar field is

$$
\begin{aligned}
& V(\psi)=\frac{1}{3} \rho_{\Lambda} c^{2}\left(\cosh ^{2} \psi+2\right) \quad(\psi \leq 0), \\
& \left(\frac{a_{3}}{a}\right)^{2}=-\sinh \psi, \quad \psi=\left(\frac{8 \pi G}{c^{2}}\right)^{1 / 2} \phi .
\end{aligned}
$$

For $t \rightarrow 0$, using (58), we get

$$
\psi \simeq \ln \left(\frac{t}{t_{\Lambda}}\right)+\left(\frac{1}{2}\right) \ln \left(\frac{3 \pi}{2}\right)+\ln \left(\frac{4}{3}\right) .
$$

For $t \rightarrow+\infty$, using (55) with a prefactor $1 / \sqrt{2}$, we get

$$
\psi \sim-2 e^{-(32 \pi / 3)^{1 / 2} t / t_{\Lambda}} .
$$

More generally, using (B.1), the evolution of the scalar field is given by

$$
\psi=-\sinh ^{-1}\left[\frac{1}{\sinh \left(\sqrt{(32 \pi / 3)}\left(t / t_{\Lambda}\right)\right)}\right] .
$$

For the quadratic equation of state (63), the density is related to the scale factor by (64). If we neglect the contribution of matter, it is possible to solve the Friedmann equation (17) with the density-radius relation (64) analytically. Introducing $R=a / a_{1}$ and $\lambda=\rho_{\Lambda} / \rho_{P} \ll 1$, we obtain

$$
\int \frac{\sqrt{1+R^{4}}}{R \sqrt{1+\lambda R^{4}}} d R=\left(\frac{8 \pi}{3}\right)^{1 / 2} \frac{t}{t_{P}}+C,
$$

which can be integrated into

$$
\begin{aligned}
& \frac{1}{\sqrt{\lambda}} \ln \left[1+2 \lambda R^{4}+2 \sqrt{\lambda\left(1+R^{4}+\lambda R^{8}\right)}\right] \\
& -\ln \left[\frac{2+R^{4}+2 \sqrt{1+R^{4}+\lambda R^{8}}}{R^{4}}\right]=4\left(\frac{8 \pi}{3}\right)^{1 / 2} \frac{t}{t_{P}}+C,
\end{aligned}
$$

where the constant $C$ is determined such that $a=l_{P}$ at $t=0$. This solution is interesting mathematically because it describes analytically a phase of early inflation and a phase of late inflation connected by a power-law evolution corresponding to the radiation. However, since (B.7) does not take into account the contribution of baryonic matter and dark matter, the intermediate phase is unphysical: the solution (B.7) describes a universe without matter! The correct solution is obtained by solving the complete Friedmann equation (100) as in Section 5.5.

\section{References}

[1] J. Binney and S. Tremaine, Galactic Dynamics, Princeton University Press, 2008.

[2] A. H. Guth, "Inflationary universe: a possible solution to the horizon and flatness problems," Physical Review D, vol. 23, pp. 347-356, 1981.
[3] A. D. Linde, "A new inflationary universe scenario: a possible solution of the horizon, flatness, homogeneity, isotropy and primordial monopole problems," Physics Letters B, vol. 108, no. 6, pp. 389-393, 1982.

[4] A. Albrecht, P. J. Steinhardt, M. S. Turner, and F. Wilczek, "Reheating an inflationary universe," Physical Review Letters, vol. 48, no. 20, pp. 1437-1440, 1982.

[5] A. Linde, Particle Physics and Inflationary Cosmology, Harwood, Chur, Switzerland, 1990.

[6] S. Weinberg, Gravitation and Cosmology, John Wiley \& Sons, 1972.

[7] E. J. Copeland, M. Sami, and S. Tsujikawa, "Dynamics of dark energy," International Journal of Modern Physics D, vol. 15, no. 11, pp. 1753-1935, 2006.

[8] A. G. Riess, A. V. Filippenko, P. Challis et al., "Observational evidence from supernovae for an accelerating universe and a cosmological constant," The Astronomical Journal, vol. 116, no. 3, p. 1009, 1998.

[9] S. Perlmutter, G. Aldering, G. Goldhaber et al., "Measurements of $\Omega$ and $\Lambda$ from 42 High-Redshift Supernovae," The Astrophysical Journal, vol. 517, no. 2, pp. 565-586, 1999.

[10] P. de Bernardis, P. A. R. Ade, J. J. Bock et al., "A flat Universe from high-resolution maps of the cosmic microwave background radiation," Nature, vol. 404, pp. 955-959, 2000.

[11] S. Hanany, P. Ade, A. Balbi et al., "MAXIMA-1: a measurement of the cosmic microwave background anisotropy on angular scales of $10^{\prime}-5^{\circ}$," The Astrophysical Journal Letters, vol. 545, no. 1, pp. L5-L9, 2000.

[12] B. Ratra and J. Peebles, "Cosmological consequences of a rolling homogeneous scalar field," Physical Review D, vol. 37, no. 12, pp. 3406-3427, 1988.

[13] A. A. Starobinsky, "How to determine an effective potential for a variable cosmological term," Journal of Experimental and Theoretical Physics Letters, vol. 68, no. 10, pp. 757-763, 1998.

[14] R. R. Caldwell, R. Dave, and P. J. Steinhardt, "Cosmological imprint of an energy component with general equation of state," Physical Review Letters, vol. 80, pp. 1582-1585, 1998.

[15] Ph. Brax and J. Martin, "Quintessence and supergravity," Physics Letters B, vol. 468, no. 1-2, pp. 40-45, 1999.

[16] A. Albrecht and C. Skordis, "Phenomenology of a realistic accelerating universe using only Planck-scale physics," Physical Review Letters, vol. 84, pp. 2076-2079, 2000.

[17] T. Barreiro, E. J. Copeland, and N. J. Nunes, "Quintessence arising from exponential potentials," Physical Review D, vol. 61, no. 12, Article ID 127301, 4 pages, 2000.

[18] L. A. Ureña-López and T. Matos, "New cosmological tracker solution for quintessence," Physical Review D, vol. 62, Article ID 081302, 4 pages, 2000.

[19] P. Brax and J. Martin, "Robustness of quintessence," Physical Review D, vol. 61, no. 10, Article ID 103502, 14 pages, 2000.

[20] T. D. Saini, S. Raychaudhury, V. Sahni, and A. A. Starobinsky, "Reconstructing the cosmic equation of state from supernova distances," Physical Review Letters, vol. 85, no. 6, pp. 1162-1165, 2000.

[21] V. Sahni and A. A. Starobinsky, "The case for a positive cosmological $\Lambda$-term," International Journal of Modern Physics D, vol. 9, no. 4, pp. 373-443, 2000.

[22] V. Sahni, "The cosmological constant problem and quintessence," Classical and Quantum Gravity, vol. 19, no. 13, pp. 34353448, 2002. 
[23] M. Pavlov, C. Rubano, M. Sazhin, and P. Scudellaro, "Analysis of two quintessence models with Type Ia supernova data," Astrophysical Journal Letters, vol. 566, no. 2, pp. 619-622, 2002.

[24] V. Sahni, T. D. Saini, A. A. Starobinsky, and U. Alam, "Statefinder-a new geometrical diagnostic of dark energy," Journal of Experimental and Theoretical Physics Letters, vol. 77, no. 5, pp. 201-206, 2003.

[25] A. Kamenshchik, U. Moschella, and V. Pasquier, "An alternative to quintessence," Physics Letters B, vol. 511, no. 2-4, pp. 265-268, 2001.

[26] N. Bilic, G. B. Tuper, and R. Viollier, "Unification of dark matter and dark energy: the inhomogeneous Chaplygin gas," Physics Letters B, vol. 535, pp. 17-21, 2002.

[27] J. S. Fabris, S. V. Goncalves, and P. E. de Souza, "Letter: density perturbations in a universe dominated by the Chaplygin gas," General Relativity and Gravitation, vol. 34, no. 1, pp. 53-63, 2002.

[28] M. C. Bento, O. Bertolami, and A. A. Sen, "Generalized Chaplygin gas, accelerated expansion, and dark-energy-matter unification," Physical Review D, vol. 66, no. 4, Article ID 043507, 5 pages, 2002.

[29] H. B. Benaoum, "Accelerated universe from modified Chaplygin gas and Tachyonic fluid," submitted, http://arxiv.org/abs/hepth/0205140.

[30] V. Gorini, A. Kamenshchik, and U. Moschella, "Can the Chaplygin gas be a plausible model for dark energy?" Physical Review $D$, vol. 67, no. 6, Article ID 063509, 2003.

[31] M. C. Bento, O. Bertolami, and A. A. Sen, "Revival of the unified dark energy-dark matter model?" Physical Review D, vol. 70, no. 8, Article ID 083519, 7 pages, 2004.

[32] U. Debnath, A. Banerjee, and S. Chakraborty, "Role of modified Chaplygin gas in accelerated universe," Classical and Quantum Gravity, vol. 21, no. 23, pp. 5609-5617, 2004.

[33] K. Karami, S. Ghaari, and J. Fehri, "Interacting polytropic gas model of phantom dark energy in non-flat universe," The European Physical Journal C, vol. 64, no. 1, pp. 85-88, 2009.

[34] P. H. Chavanis, "Models of universe with a polytropic equation of state: I. The early universe," submitted, http://arxiv.org/abs/ 1208.0797.

[35] P. H. Chavanis, "Models of universe with a polytropic equation of state: II. The late universe," submitted, http://arxiv.org/abs/ 1208.0801.

[36] P. H. Chavanis, "Models of universe with a polytropic equation of state: III. The phantom universe," submitted, http://arxiv.org/ abs/1208.1185.

[37] H. B. Sandvik, M. Tegmark, M. Zaldarriaga, and I. Waga, "The end of unified dark matter?” Physical Review D, vol. 69, no. 12, Article ID 123524, 7 pages, 2004.

[38] S. Weinberg, "The cosmological constant problem," Reviews of Modern Physics, vol. 61, no. 1, pp. 1-23, 1989.

[39] T. Padmanabhan, Theoretical Astrophysics, vol. 3 of Galaxies and Cosmology, Cambridge University Press, 2002.

[40] R. R. Caldwell, "A phantom menace? Cosmological consequences of a dark energy component with super-negative equation of state," Physics Letters B, vol. 545, pp. 23-29, 2002.

[41] R. R. Caldwell, M. Kamionkowski, and N. N. Weinberg, "Phantom energy: dark energy with $w<-1$ causes a cosmic doomsday," Physical Review Letters, vol. 91, no. 7, Article ID 071301, 4 pages, 2003.

[42] B. McInnes, "The dS/CFT correspondence and the big smash," Journal of High Energy Physics, vol. 2002, no. 8, p. 29, 2002.
[43] S. M. Carroll, M. Hoffman, and M. Trodden, "Can the dark energy equation-of-state parameter $w$ be less than -1?" Physical Review D, vol. 68, no. 2, Article ID 023509, 11 pages, 2003.

[44] P. Singh, M. Sami, and N. Dadhich, "Cosmological dynamics of a phantom field," Physical Review D, vol. 68, Article ID 023522, 7 pages, 2003.

[45] J. M. Cline, S. Jeon, and G. D. Moore, “The phantom menaced: constraints on low-energy effective ghosts," Physical Review D, vol. 70, no. 4, Article ID 043543, 4 pages, 2004.

[46] M. Sami and A. Toporensky, "Phantom field and the fate of the universe," Modern Physics Letters A, vol. 19, no. 20, pp. 1509-1517, 2004.

[47] S. Nesseris and L. Perivolaropoulos, "Fate of bound systems in phantom and quintessence cosmologies," Physical Review D, vol. 70, no. 12, Article ID 123529, 9 pages, 2004.

[48] E. Babichev, V. Dokuchaev, and Y. Eroshenko, "Black hole mass decreasing due to phantom energy accretion," Physical Review Letters, vol. 93, no. 2, Article ID 021102, 4 pages, 2004.

[49] P. F. González-Díaz and C. L. Sigüenza, "The fate of black holes in an accelerating universe," Physics Letters B, vol. 589, pp. 7882, 2004.

[50] P. F. González-Díaz and C. L. Sigüenza, "Phantom thermodynamics," Nuclear Physics B, vol. 697, pp. 363-386, 2004.

[51] S. Nojiri and S. D. Odintsov, "Final state and thermodynamics of a dark energy universe," Physical Review D, vol. 70, no. 10, Article ID 103522, 15 pages, 2004.

[52] S. Nojiri, S. D. Odintsov, and S. Tsujikawa, "Properties of singularities in the (phantom) dark energy universe," Physical Review D, vol. 71, no. 6, Article ID 063004, 16 pages, 2005.

[53] H. Štefančić, "Expansion around the vacuum equation of state: sudden future singularities and asymptotic behavior," Physical Review D, vol. 71, no. 8, Article ID 084024, 9 pages, 2005.

[54] M. Bouhmadi-López, P. F. González-Díaz, and P. Martín-Moruno, "Worse than a big rip?" Physics Letters B, vol. 659, pp. 1-5, 2008.

[55] H. García-Compeán, G. García-Jiménez, O. Obregón, and C. Ramírez, "Crossing the phantom divide in an interacting generalized Chaplygin gas," Journal of Cosmology and Astroparticle Physics, vol. 2008, no. 7, p. 16, 2008.

[56] L. Fernández-Jambrina, “w-cosmological singularities,” Physical Review D, vol. 82, Article ID 124004, 5 pages, 2010.

[57] P. H. Frampton, K. J. Ludwick, and R. J. Scherrer, “The little rip," Physical Review D, vol. 84, Article ID 063003, 5 pages, 2011.

[58] P. Steinhardt, "Cosmological challenges for the 21st century," in Critical Problems in Physics, V. L. Fitch and D. R. Marlow, Eds., Princeton University Press, Princeton, NJ, USA, 1997.

[59] I. Zlatev, L. Wang, and P. J. Steinhardt, "Quintessence, cosmic coincidence, and the cosmological constant," Physical Review Letters, vol. 82, no. 5, pp. 896-899, 1999.

[60] L. Amendola and D. Tocchini-Valentini, "Stationary dark energy: the present universe as a global attractor," Physical Review $D$, vol. 64, no. 4, Article ID 043509, 5 pages, 2001.

[61] L. P. Chimento, A. S. Jakubi, and D. Pavón, "Dark energy, dissipation, and the coincidence problem," Physical Review D, vol. 67, no. 8, Article ID 087302, 3 pages, 2003.

[62] G. A. Monerat, G. Oliveira-Neto, E. V. Corrêa Silva et al., "Dynamics of the early universe and the initial conditions for inflation in a model with radiation and a Chaplygin gas," Physical Review D, vol. 76, no. 2, Article ID 024017, 11 pages, 2007. 
[63] C. M. Muller, "Cosmological bounds on the equation of state of dark matter," Physical Review D, vol. 71, no. 4, Article ID 047302 , 4 pages, 2005.

[64] T. Harko, "Evolution of cosmological perturbations in BoseEinstein condensate dark matter," Monthly Notices of the Royal Astronomical Society, vol. 413, pp. 3095-3104, 2011.

[65] P. H. Chavanis, "Growth of perturbations in an expanding universe with Bose-Einstein condensate dark matter," Astronomy \& Astrophysics, vol. 537, article A127, 2012. 

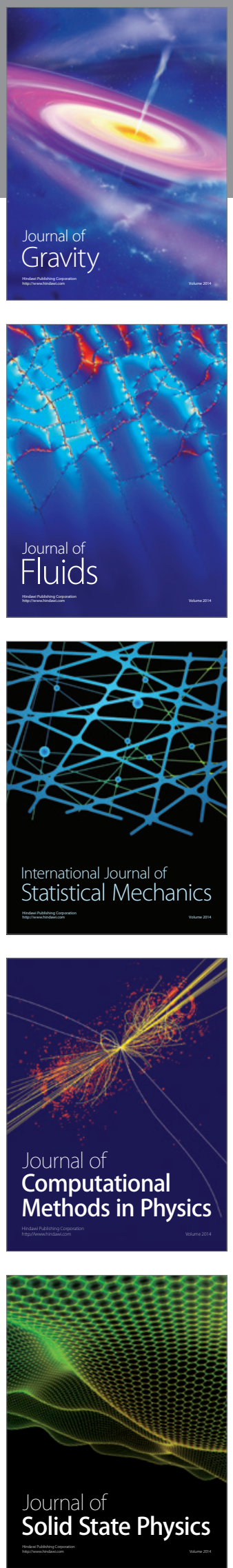

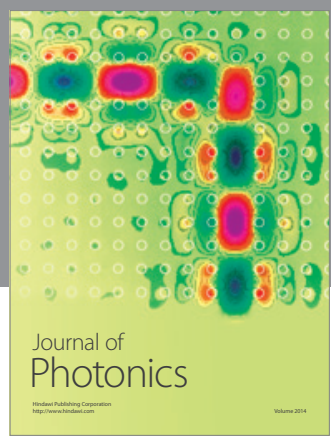

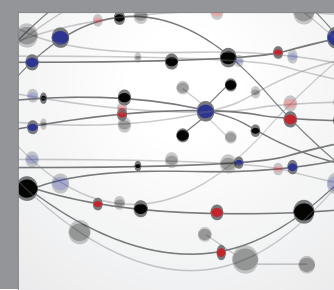

The Scientific World Journal

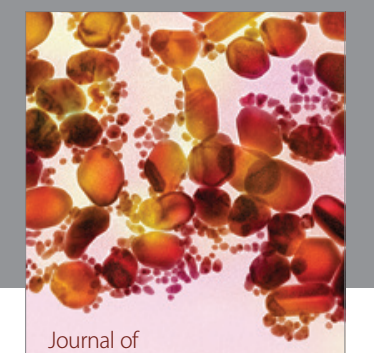

Soft Matter
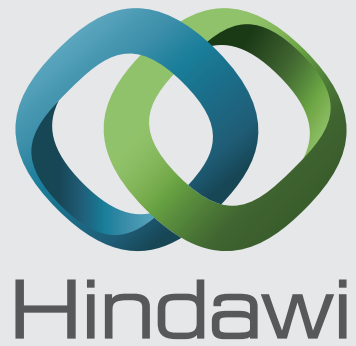

Submit your manuscripts at

http://www.hindawi.com
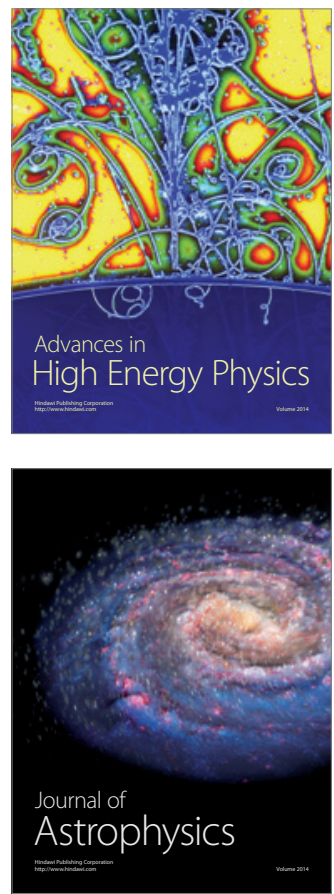
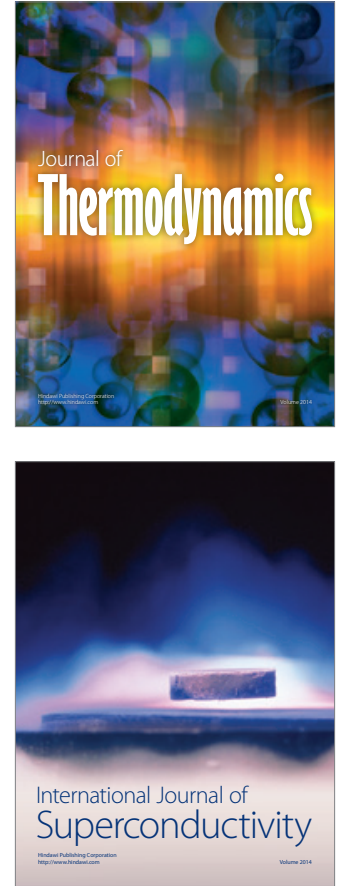
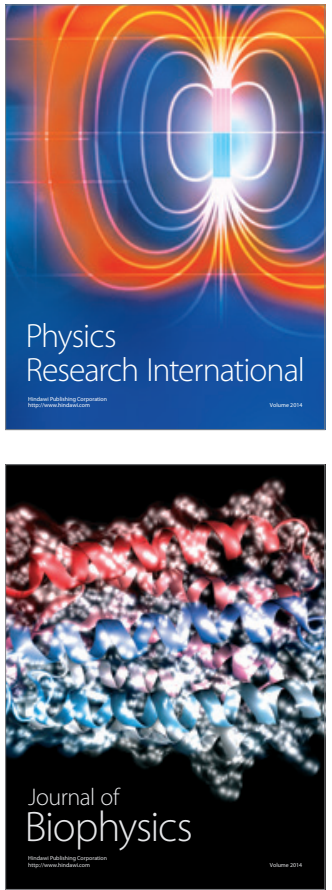
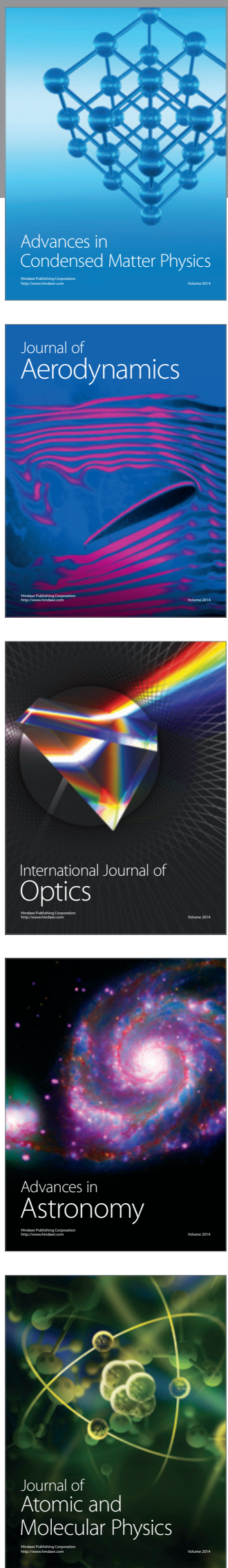